\title{
Antigen receptor locus dynamics is orchestrated near the sol-gel phase transition to enforce stepwise VDJ gene rearrangement
}

Nimish Khanna ${ }^{1,4}$, Yaojun Zhang ${ }^{2,4}$, Joseph S. Lucas ${ }^{1}$, Olga K. Dudko ${ }^{3}$ \& Cornelis Murre ${ }^{1}$

${ }^{1}$ Division of Biological Sciences, 0377

Department of Molecular Biology

University of California, San Diego

La Jolla, CA 92093

${ }^{2}$ Princeton Center for Theoretical Science

Princeton University

Princeton, NJ 08544

${ }^{3}$ Department of Physics

University of California, San Diego

La Jolla, CA 92093

${ }^{4}$ These authors made equal contributions

e-mail: dudko@physics.ucsd.edu; murre@biomail.ucsd.edu 
Diverse antibody repertoires are generated through remote genomic interactions involving immunoglobulin variable $\left(V_{H}\right)$, diversity $\left(D_{H}\right)$ and joining $\left(J_{H}\right)$ gene segments. How such interactions are orchestrated remains unknown. We developed a novel strategy to track $V_{H}-D_{H} J_{H}$ motion and interactions in live B-lymphocytes. We found that $V_{H}$ and $D_{H} J_{H}$ segments were trapped in configurations that only allowed constrained local motion, such that spatially proximal $V_{H}$ and $D_{H} J_{H}$ segments remained in proximity, whereas spatially remote segments explored their immediate neighborhood while remaining remote. Comparison of experimental and simulated data revealed that such a highly constrained motion was imposed by a network of cross-linked chromatin chains characteristic of a gel phase, yet it was poised near the sol phase, a solution of independent chromatin chains. We propose that epigenetically induced gel droplets and the proximity to the sol-gel phase transition constitute the mechanism that orchestrates ordered VDJ rearrangement. 
It is now well established that the mammalian genome, although dynamic, is highly structured. Chromosomes occupy individual territories and interact sparsely. Within chromosomes, chromatin loop domains are anchored by CTCF proteins and serve as the fundamental units of chromosome structure . $^{1,2}$. The folding of the loop domains is orchestrated by members of the cohesin complex, whereby cohesin extrudes loops of chromatin in a progressive fashion until a pair of convergent CTCF sites is reached ${ }^{3}$. The chromatin fiber is also subject to large-scale epigenetic modifications that include DNA methylation, chromatin remodelers, histone modifications and non-coding transcription. Recent studies have proposed that weak multivalent interactions result in phase-separated regions that compartmentalize gene expression ${ }^{4}$

The immunoglobulin heavy chain (Igh) locus is assembled through long-range genomic interactions involving variable $\left(\mathrm{V}_{H}\right)$, diversity $\left(\mathrm{D}_{H}\right)$, joining $\left(\mathrm{J}_{H}\right)$, and constant $\left(\mathrm{C}_{H}\right)$ coding elements ${ }^{5}$. Two endonucleases, RAG1 and RAG2, initiate the recombination reaction by generating double-strand DNA breaks in the recombination signal sequences that flank the $V_{H}, D_{H}$ and $J_{H}$ segments ${ }^{6,7}$. The variable regions are segregated into two distinct clusters, the distal and proximal $\mathrm{V}_{\mathrm{H}}$ regions, that collectively span approximately 2.7 Mb. The $\mathrm{D}_{\mathrm{H}}$ and $\mathrm{J}_{\mathrm{H}}$ elements are separated from each other by $50 \mathrm{~kb}$ and are located upstream of the intronic enhancer $E \mu$. Located downstream of $D_{H}$ and $J_{H}$ are the constant regions as well as a cluster of CTCF sites named the Igh 3' regulatory region, also known as the Igh super-anchor ${ }^{5,8}$. The $\mathrm{V}_{\mathrm{H}}$ regions are associated with CTCF binding sites, which are positioned in a convergent orientation towards the super-anchor 8,9 . Two CTCF sites separate the $V_{H}$ from the $D_{H} J_{H}$ elements to suppress predominant rearrangement of the proximal $V_{H}$ region cluster and to orchestrate ordered lgh locus rearrangement ${ }^{9,10,11}$. Prior to $V_{H}-D_{H} J_{H}$ rearrangement, the $D_{H} J_{H}$ region folds into a singular loop domain that insulates RAG activity across loop domains ${ }^{12}$.

The Igh locus is also characterized by a large spectrum of epigenetic modifications distributed across the $V_{H}$ and $D_{H} J_{H}$ regions ${ }^{13}$. A subset of these modifications is deposited in a lineage- and developmental stage-specific fashion and correlate with ordered lgh $V_{H^{-}} D_{H} J_{H}$ rearrangement ${ }^{14}$. Prior to $D_{H} J_{H}$ rearrangement, non-coding transcription is initiated, and histone marks such as H3K27Ac, H3Ac and 
H3K4me1 are deposited within the $D_{H} J_{H}$ loop domain ${ }^{15}$. The deposition of these marks is controlled, at least in part, by an intronic enhancer located immediately downstream of the $D_{H} J_{H}$ region ${ }^{13}$. $V_{H^{-}}-D_{H} J_{H}$ rearrangement is also associated with the deposition of epigenetic marks across the $\mathrm{V}_{\mathrm{H}}$ regions that include $\mathrm{H} 3 \mathrm{Ac}, \mathrm{H} 3 \mathrm{~K} 27 \mathrm{me} 3, \mathrm{H} 3 \mathrm{~K} 4 \mathrm{me} 1$ and $\mathrm{H} 3 \mathrm{~K} 27 \mathrm{me} 3^{16}$. Finally, the chromatin remodeler BRG1 binds to multiple sites to assemble crosslinks across the Igh locus chromatin fiber, leading to lgh locus contraction and distal $\lg \mathrm{V}_{\mathrm{H}^{-}} \mathrm{D}_{\mathrm{H}} \mathrm{J}_{\mathrm{H}}$ rearrangement ${ }^{17}$.

Recent studies using a single-color labeling strategy demonstrated that $V_{H}$ and $D_{H} J_{H}$ elements undergo anomalous diffusion in a viscoelastic environment whereby they bounce back and forth in a spring-like fashion ${ }^{18,19}$. However, since only a single element was marked, these studies did not permit the visualization and description of $V_{H}-D_{H} J_{H}$ genomic interactions and $V_{H}-D_{H} J_{H}$ motion in live $B$ cells. Here, we designed and generated tandem arrays of wild-type TET-operator and mutant TET-operator binding sites to separately mark both the $V_{H}$ and the $D_{H} J_{H}$ elements. We found that the lgh locus adopted a wide spectrum of configurations that were dynamic enough to allow local motion yet stable enough to provide a strong long-term confinement. To determine the mechanistic origin of this confined motion, we performed Molecular Dynamics simulations of a hierarchy of polymer models. We found that that the lgh loop domains are organized as gel droplets poised near a sol-gel phase transition. We propose that the proximity of the Igh locus to the sol-gel phase boundary provides a tradeoff between stability and responsiveness: the ordered nature of the crosslinked network, formed by the locus and characteristic of a gel, enables ordered lgh locus rearrangement by facilitating and insulating $V_{H^{-}} D_{H} J_{H}$ and $D_{H^{-}} J_{H}$ interactions, while the disordered nature of a solution of independent chains, characteristic of a sol, permits randomness in $\mathrm{V}_{\mathrm{H}^{-}} \mathrm{D}_{\mathrm{H}} \mathrm{J}_{\mathrm{H}}$ encounters and rapid Igh locus re-assembly.

\section{Results}

An approach to monitoring genomic interactions in live cells. In previous studies we tracked either $V_{H}$ or $D_{H} J_{H}$ motion in live pro-B cells using tandem arrays of TET-operator binding sites ${ }^{19}$. We next aimed to monitor genomic encounters directly in live cells using a dual labeling approach (Fig. 1a). Attempts to 
insert tandem arrays of LAC operators in conjunction with TET-operator binding sites failed because tandem arrays of LAC operator binding sites were unstable in expanding pro-B cell cultures (data not shown). We therefore turned to a new strategy to simultaneously track the motion of paired genomic elements. Specifically, we used wild-type and mutant TET-repressor binding sites associated with unique DNA binding specificities ${ }^{20,21}$. From these, we selected a mutant TET repressor (4C5G) carrying two amino acid substitutions (E37A and P39K) in the TET-DNA binding domain since it showed nonoverlapping DNA binding activity (Fig. 1b). Tandem arrays containing 320 copies of the mutant TET 4C5G binding site (CCGTCAGTGACGG) were constructed essentially as described previously (Fig. 1c) ${ }^{22}$.

In principle, two distinct repressor modules should permit the simultaneous tracking of two genomic regions. However, TET repressor binds as dimers and heterodimers of wild-type TET, and mutant TET protomers, which would interfere with the ability to bind tandem arrays of either WT-TET or MUT-TET binding sites specifically and selectively. To overcome this obstacle, we converted the MUT-TET repressor dimer into a single chain using a 29 amino acid linker. The single chain (sc) TET-repressor binds to its cognate binding site as a monomer ${ }^{21}$. To generate two differentially labeled fusion proteins, wild-type TET-repressor was fused to GFP (Fig. 1d). The mutant (sc) TET (4C5G) repressor was tagged with a polypeptide (SNAP-TAG) that has the ability to bind cell-permeable fluorescent dyes (Fig. 1d). To boost expression of the wild-type and mutant TET-repressors, both proteins were codon-optimized. Finally, to optimize the signal-to-background fluorescence ratio, the TET-SNAP-tag repressor was placed under control of a mutant Kozak sequence (GCCTCCATG) to damp translation and reduce background fluorescence (Fig. 1d).

Tracking $V_{H}-D_{H} J_{H}$ motion and $V_{H}-D_{H} J_{H}$ encounters in live B-lymphocytes. In previous studies we have generated mice that carried tandem arrays of WT-TET operator binding sites in either the $V_{H}$ or $D_{H} J_{H}$ regions ${ }^{19}$. From mice harboring a tandem array of WT-TET binding sites adjacent to the $D_{H} J_{H}$ region we derived immortalized RAG-deficient pro-B cell lines. Briefly, RAG1-deficient WT-TET $D_{H} J_{H}$ pro-B cells 
were isolated, grown in the presence of IL7 and SCF, transduced with BCR-ABL virus and cultured in methylcellulose-containing media. After seven days, colonies appeared that were expanded in the presence of S17 feeder cells, IL7 and SCF. This approach yielded BCR-ABL-transformed pro-B cell lines carrying a tandem array of TET-operator binding sites that marked the $D_{H} J_{H}$ region (Fig. 1a). Next, we inserted a tandem array of mutant TET repressor binding sites within the $V_{H}$ region cluster. $A$ template containing 320 copies of the mutant TET 4C5G binding site (CCGTCAGTGACGG) was constructed (Fig. 1c). The tandem array was next inserted into the $V_{H}$ 8-3 region of $D_{H} J_{H}$ WT-TET pro-B cells using CAS9CRISPR engineering. Stable clones were isolated using blasticidin as a selectable marker, expanded and examined for proper insertion into the genome using PCR and the appropriate. Two clones were isolated that contained the tandem array of MUT-TET binding sites inserted into the $\mathrm{V}_{\mathrm{H}}$ region, expanded and transduced with virus expressing WT-TET GFP and MUT-TET SNAP-TAG (Fig. 1d). Two days posttransduction, the cells were immobilized on poly-lysine coated dishes, incubated with the cell-permeable dye TMR-STAR to label MUT-TET SNAP-TAG bound sites and imaged using the OMX microscope platform. Cells transduced with virus expressing WT-GFP revealed two fluorescent foci consistent with a tandem array of WT-TET binding sites inserted near the $D_{H} J_{H}$ region on both alleles (Fig. 2a; left panels). Cells transduced with virus expressing MUT-TET SNAP tag revealed a singular fluorescent center consistent with the insertion of a MUT-TET tandem array in the distal $\mathrm{V}_{\mathrm{H}}$ region on a single allele (Fig. 2a). Transduced $V_{H}$ MUT-TET; $D_{H} J_{H}$ WT-TET pro-B cells were imaged every 2 seconds for 400 seconds in short term imaging or every 40 seconds for 4800 seconds (Supplementary Movies 1 and 2).

To validate the experimental strategy using wild-type and mutant operator sites as a means to track long-range genomic interactions, we compared the distribution of the spatial distances separating the $V_{H}$ and $D_{H} J_{H}$ elements, obtained by tracking $V_{H^{-}} D_{H} J_{H}$ motion in cells carrying WT-TET and MUT-TET binding sites, to those obtained by 3D-FISH measurements (Fig. 2b). We found that both methods yielded similar distributions, consistent with the insertion of tandem arrays of WT-TET and MUT-TET binding sites into the $V_{H}$ and $D_{H} J_{H}$ regions, respectively (Fig. 2c). To determine the localization error associated with 
marking paired genomic regions with fluorescently labeled wild-type and mutant TET proteins, pro-B cells harboring tandem arrays of MUT-TET binding sites were transduced with virus expressing MUT-TET GFP (green fluorescence) and virus expressing MUT-TET SNAP-TAG (red fluorescence) (Fig. 2d). Cells that showed overlapping green and red fluorescent foci were imaged every 2 seconds for 400 seconds. The positions of the two fluorescent foci as a function of time were recorded to determine localization error. We found mean-squared displacement due to the measurement error to be approximately $0.02 \mu \mathrm{m}^{2}$, or an average localization error of $22 \mathrm{~nm}$ for the $x$ - and $y$-coordinates and $95 \mathrm{~nm}$ for the $z$-coordinate for each marker (Supplementary Information). To extract the true $\mathrm{V}_{\mathrm{H}}-\mathrm{D}_{\mathrm{H}} \mathrm{J}_{\mathrm{H}}$ genomic motion from the observed apparent motion, the rotational and translational nuclear motion as well as localization error were eliminated using two independent procedures (Supplementary Information), neither one involving any adjustable parameters and both found to yield very similar outcomes (Supplementary Fig. 1). Taken together, these results showed that, by using tandem arrays of wild-type and mutant TET operator binding sites to mark coding or regulatory DNA elements, paired remote genomic interactions can be tracked with relatively high accuracy.

$V_{H}-D_{H} J_{H}$ trajectories reveal highly constrained, local motion. The individual trajectories of the spatial distances separating the labeled $V_{H}$ and $D_{H} J_{H}$ regions revealed that $V_{H}-D_{H} J_{H}$ motion was extremely confined: the $V_{H}$ and $D_{H} J_{H}$ segments that were initially in close spatial proximity remained in proximity, whereas the segments that were initially spatially remote remained remote (Fig. 3a and Supplementary Fig. 2). The corresponding probability distribution of $V_{H}-D_{H} J_{H}$ distances generated from these trajectories covered a broad range of distances $(0.2-1.2 \mu \mathrm{m})$ suggesting that, across the population of cells, the locus adopts a wide spectrum of chromatin configurations (Fig. 3a; right panel). The distribution of $V_{H^{-}} D_{H} J_{H}$ distances exhibited bimodality that persisted for at least 400 seconds, indicating at least two dominant types of Igh locus configurations. Color-coding the trajectories of $\mathrm{V}_{\mathrm{H}}-\mathrm{D}_{\mathrm{H}} \mathrm{J}_{\mathrm{H}}$ spatial distances according to their mean values revealed an intriguing de-mixing effect: the distances within the individual pairs fluctuated around respective mean values that remained nearly constant, together resulting in a "rainbow" 
pattern that persisted for at least an hour (Fig. 3a, left panel, and Supplementary Fig. 2). These data indicate that the Igh locus adopts a wide spectrum of configurations that are sufficiently dynamic to allow local motion, yet stable enough to provide a long-term ( $>60$ minutes) confinement that either co-localizes $V_{H}$ and $D_{H} J_{H}$ gene segments or isolates them from each other.

The properties of $\mathrm{V}_{\mathrm{H}}-\mathrm{D}_{\mathrm{H}} \mathrm{J}_{\mathrm{H}}$ motion averaged across the trajectories were examined by computing the mean squared displacements (MSD) and the velocity autocorrelation functions (VAF) (Fig. 3b-d). MSD and VAF were calculated using pairwise $V_{H}-D_{H} J_{H}$ and $D_{H} J_{H^{-}}-D_{H} J_{H}$ distance trajectories, which eliminated the effect of nuclear motion. Time-averaged and time-and-ensemble-averaged MSD exhibited a subdiffusive scaling exponent $(\alpha<1)$ both for intrachromosomal $V_{H^{-}} D_{H} J_{H}$ and interchromosomal $D_{H} J_{H^{-}}$ $D_{H} J_{H}$ motion (Fig. 3b and 3c, Supplementary Fig. 3 and Supplementary Table 1). Specifically, $V_{H^{-}} D_{H} J_{H}$ motion was found to be extremely subdiffusive, characterized by a scaling exponent a that decreased from 0.35 at short time scales down to 0.2 at long time scales (Fig. 3c and Supplementary Table 1). VAFs exhibited negative correlations indicative of a push-back from the environment (Fig. 3d). For the intrachromosomal motion, the value of VAF at the dip approached the theoretical limit $(-0.5)$ of an extreme confinement (Fig. 3d). The collapse of the velocity autocorrelation curves upon rescaling of the time axis indicated that the motion was self-similar, i.e. exhibited similar patterns on different spatial and temporal scales (Fig. $3 d$, right panel). Taken together, these data indicate that $V_{H}-D_{H} J_{H}$ motion is extremely subdiffusive as the result of a viscoelastic and spatially confined environment.

Global confinement from chromatin loops eliminates bias in variable region usage. To explore the mechanistic origin of the viscoelasticity and confinement that govern $V_{H}-D_{H} J_{H}$ motion, we modeled the chromatin fiber as a bead-spring polymer chain using Molecular Dynamics (MD) simulations (Fig. 4$)^{24}$. Given the large number of molecular components and an extensive parameter space that could in principle be incorporated in the simulations, we constrained the model by utilizing multiple independent sets of experimental data. As the first step, we sought to identify the minimal model that could reproduce 
the structural properties of the lgh locus, namely the plateau in the $V_{H}-D_{H} J_{H}$ spatial distances as a function of the genomic distance observed in $3 \mathrm{D} \mathrm{FISH}{ }^{25,26}$. As the second step, which is described in the next section, the resulting minimal model was refined such that it could, in addition, reproduce the dynamic properties of the locus, namely the de-mixing of the $V_{H^{-}} D_{H} J_{H}$ distances and the strongly subdiffusive scaling exponent.

To capture the structural properties of the Igh locus, we simulated a hierarchy of worm-like chain polymer models reflecting chromatin configurations of increasing complexity: an unstructured chromatin chain, a single loop configuration, a two-loop configuration, and a chain enclosed in a confinement that mimicked a multiple-loop configuration averaged over many realizations of different ways of looping (Fig. 4a). As expected, the unstructured chain model failed to generate the plateau in the spatial versus genomic distances, instead producing a monotonically increasing dependence (Fig. 4a). A spatial confinement enclosing the chain was necessary to achieve the saturation of the spatial distance at $0.4 \mu \mathrm{m}$ over more than $2 \mathrm{Mb}$ of genomic distances as observed in 3D-FISH experiments ${ }^{25,26}$. Similarly, the average over a large number of explicit multiple-loop configurations in the absence of confinement also reproduced the plateau (Supplementary Fig. 4). These results indicate that chromatin loops act as a primary source of large-scale confinement, which enables $\mathrm{V}_{\mathrm{H}}$ regions that are spread over vast genomic distances to reach the $D_{H} J_{H}$ region with similar frequencies, independent of their genomic proximity to the $\mathrm{D}_{\mathrm{H}} \mathrm{J}_{\mathrm{H}}$ region.

Immunoglobulin heavy chain locus in a gel-like state. While the model of the lgh locus compartmentalized as loop domains correctly reproduced the structural properties (Fig. 4a), it yielded intersecting dynamic trajectories (Fig. 4c, left panel) that were inconsistent with the de-mixed trajectories observed in live-cell imaging (Fig, 3a and Supplementary Fig. 2). This discrepancy suggested yet another layer of constraint, which confined the motion locally. A plausible mechanism of such a local confinement is the cross-bridging of the chromatin fiber via cross-links, analogous to the mechanism that was recently 
proposed for the formation of super-enhancers' whereby cross-links induce phase separated condensates $^{4}$. To examine a potential role of cross-links as the source of local confinement, we introduced in the simulations $5 \%$ cross-linkable sites across the Igh locus which could undergo pairwise binding and unbinding with tunable kinetics (Fig. 4b; Supplementary Information). Notably, in the presence of crosslinks, the de-mixing of the trajectories was recovered and became progressively pronounced upon increasing cross-link residence time (Fig. 4c).

In polymer physics, a solution of free (un-crosslinked) chains and a network of cross-linked chains are known to represent two distinct physical phases: a sol phase and a gel phase, respectively ${ }^{27}$. The modeling approach described above and guided by the experimental observations both at the populationand single trajectory-levels suggested that the phase state of the lgh locus corresponds to a gel, or a dynamic three-dimensional network of chromatin chains and associated factors, held together by crosslinks ${ }^{28}$. As a consequence of gelation, those $V_{H}$ and $D_{H} J_{H}$ segments that end up in close spatial proximity by virtue of their chromatin configuration will remain in close proximity and likely encounter each other within a short time (seconds to tens of minutes). Conversely, those $V_{H}$ and $D_{H} J_{H}$ segments that end up spatially remote as a result of their chromatin configuration will remain separated for a long time (more than an hour). Thus, gelation, by constraining the motion locally via cross-links, either facilitates or hinders genomic encounters, depending on the initial spatial separation imposed by a particular fold of the chromatin backbone.

Gel is a solid ${ }^{27}$, and thus cross-linking imparts solid-like properties to the locus, which otherwise (in the absence of crosslinks) would possess the liquid-like properties of a sol. Given that the elasticity of the solid phase and the fluidity of the liquid phase can both be advantageous from a biological prospective, we asked: to what extent are the properties of Igh locus solid-like as opposed to liquid-like? Or, equivalently, how deeply in the gel phase is the locus positioned on a phase diagram? The tuning of the bond lifetime of the simulated cross-links from irreversible bonds (strong gel) to short-lived bonds (weak gel) and further to the absence of cross-linking (sol) resulted in a systematic change in the slope (a) of the MSD (Fig. 5a and 
Supplementary Fig. 5). The bond lifetime on the order of 10 seconds was found to yield the best agreement between simulated and experimentally measured MSD, indicating that the state of the locus corresponds to a weak gel poised close to the boundary of sol and gel phases (Fig. 5a). Taken together, these results indicate that a large-scale confinement imposed by chromatin loops and a local confinement imposed by the cooperative action of cross-links together constitute the mechanistic origin of the strongly subdiffusive $\mathrm{V}_{\mathrm{H}^{-}}-\mathrm{D}_{\mathrm{H}} \mathrm{J}_{\mathrm{H}}$ motion, and that the Igh locus is found in the state of a weak gel.

$V_{H}-D_{H} J_{H}$ encounter times in a gel state. The two-color imaging strategy allowed us to measure the spatial distance within the individual $\mathrm{V}_{\mathrm{H}}-\mathrm{D}_{\mathrm{H}} \mathrm{J}_{\mathrm{H}}$ pairs as a function of time and thus enabled a direct estimation of the first-passage times (FPTs), or the waiting times of the first encounters (Fig. 5b and Supplementary Fig. 6). We set a threshold value for the distance at which the segments can be considered interacting by combining the value of $30 \mathrm{~nm}$ for the true interaction distance and the contribution from localization error (Supplementary Information). A dip in the distance below the threshold indicates a potential encounter, and the corresponding time provides an estimation for the FPT. In order to acquire a rich statistics of the encounter events, we generated a pool of STI-571 treated pro-B cells, which yielded a significantly higher fraction of potential encounters. In the sub-population of the cells in which $V_{H}$ and $D_{H} J_{H}$ segments were spatially remote at the start of imaging (initial separation $>0.55 \mu m$ ), no potential encounters were detected throughout the imaging period. In contrast, the sub-population of cells with $V_{H}$ and $D_{H} J_{H}$ segments spatially co-localized at the start of imaging $(<0.55 \mu \mathrm{m})$, the potential encounters occurred already within minutes in more than $40 \%$ of these cells (Fig. $5 \mathrm{~b}$ and Supplementary Fig. 6). To examine whether the weak-gel model of the Igh locus reproduces the experimentally observed firstencounter times, we generated the trajectories of $\mathrm{V}_{\mathrm{H}}-\mathrm{D}_{\mathrm{H}} \mathrm{J}_{\mathrm{H}}$ distances from MD simulations of a globally confined, weakly cross-linked chain. The range of the first-encounter times extracted from the simulated trajectories (Fig. 5c) was in quantitative agreement with the range observed experimentally (Fig. 5b). To investigate the dependence of the FPTs on the spatial distance, we examined both the measured and 
simulated FPTs as a function of mean spatial distance, the parameter that sets the length scale of this first-passage-time problem. The experimental and simulated data showed good quantitative agreement (Fig. 5d). Furthermore, on a log-log plot, both types of data resulted in the best fit by a line with a slope of 2/ $\alpha$ (Fig. 5d and Supplementary Fig. 7), in agreement with the predicted scaling MFPT $\sim R^{2 / a} 19$. Taken together, the first-encounter times for $\mathrm{V}_{\mathrm{H}}-\mathrm{D}_{\mathrm{H}} \mathrm{J}_{\mathrm{H}}$ interactions from live cell imaging, as well as the scaling of these times with the $V_{H}-D_{H} J_{H}$ spatial distance, were found to be in quantitative agreement with the encounter times generated by a model of the Igh locus as a cross-linked network of chromatin and associated factors that is poised near the sol/gel phase boundary.

\section{Discussion}

It is now established that the Igh locus is organized into loop domains $(1,2)$. However, our understanding of the mechanisms that govern the dynamics of $V(D) J$ rearrangement remains rudimentary at best. The approach developed here enabled us to simultaneously track the motion of $V_{H}$ and $D_{H} J_{H}$ elements and to visualize $V_{H}-D_{H} J_{H}$ encounters in live $B$ cells. The resulting findings highlight the link between the spatial and temporal aspects of Igh locus organization. Namely, the interplay between the confinement imposed by loop domains and crosslinks on the one hand and the inherently subdiffusive motion of the chromatin fiber on the other hand can either facilitate or hinder genomic interactions within the Igh locus. Indeed, in mediating genomic encounters, subdiffusive $\mathrm{V}_{\mathrm{H}}-\mathrm{D}_{\mathrm{H}} \mathrm{J}_{\mathrm{H}}$ motion has its advantages and disadvantages compared to normal diffusion: a sub-diffuser searches more efficiently at short distances due to its tendency to explore the space compactly, but it is less efficient searching over long distances due to the slower growth of its MSD with time. Spatial confinement enhances each of these two tendencies: $V_{H}$ and $D_{H} J_{H}$ segments that are brought into spatial proximity through looping and whose proximity is further reinforced by cross-links become spatially co-localized; this amplifies the efficiency of their subdiffusive search over short distances, thereby facilitating their encounters. Conversely, $V_{H}$ and $D_{H} J_{H}$ gene segments that end up in different loops, which are further stabilized by crosslinks, become isolated from each other; 
this amplifies the inefficiency of their subdiffusive search over long distances, further hindering their encounters.

Our study further revealed that the structural and dynamic properties of the Igh locus are consistent with those of a weak gel, or a network of chromatin chains and associated factors that are bridged via short-lived cross-links. We concluded that, in the phase space, the Igh locus is positioned within the gel phase (and thus it is a solid) but close to the sol/gel (or liquid/solid) phase boundary ${ }^{29}$, akin to a molded pudding with barely enough gelatin to hold its form. Given that the lifetime of cross-links in the cell is regulated by epigenetic marks, non-coding transcription and chromatin remodelers, and thus represents a dynamical quantity, our findings suggest that the gelation of chromatin is a biological process that is finely tuned with respect to a dynamical variable - the strength of the cross-links. Proximity to the liquid/solid phase boundary allows the loop domains within the Igh locus to rapidly switch their chromatin state by tuning the deposition of epigenetic marks and recruiting chromatin remodelers. Tuning the chromatin properties further into the solid phase (gel) promotes the formation of phase-separated gel droplets, which in turn facilitate the encounters between spatially close $D_{H}$ and $J_{H}$ (or $V_{H}$ and $D_{H} J_{H}$ ) segments and hinder the encounters between spatially remote segments. Conversely, tuning the chromatin state closer to the liquid phase (sol) allows the existing gel droplets to dissolve and new gel droplets to form readily, thus enabling a rapid and ordered re-assembly of the Igh locus.

On the basis of these findings, we propose that the epigenetically-controlled formation of gel droplets comprising chromatin loop domains introduces two modes in $V_{H}-D_{H} J_{H}$ and $D_{H}-J_{H}$ encounters facilitation and hindrance, thereby preventing premature rearrangements and enforcing VDJ recombination to proceed in a stepwise fashion. At the same time, the proximity of the lgh locus to the sol/gel phase boundary and the resulting propensity to switch between liquid and solid states enables a rapid re-assembly of the locus such that the VDJ recombination can readily proceed from one step to the next. Weak gelation therefore offers a tradeoff between stability and responsiveness in the generation of antigen receptor diversity. 
Finally, what is the molecular nature of the cross-links that result in gelation of the Igh locus? It has long been established that the chromatin landscape across the $V_{H}$ and $D_{H} J_{H}$ regions is developmentally regulated ${ }^{13}$. Immediately prior to $D_{H^{-}} J_{H}$ rearrangement, the $D_{H^{-}} J_{H}$ loop domain is closely associated with non-coding transcription, and histones across the $D_{H^{-}} J_{H}$ loop domain are hyperacetylated ${ }^{20,30}$. Likewise, the onset of $\mathrm{V}_{\mathrm{H}}-\mathrm{D}_{\mathrm{H}} \mathrm{J}_{\mathrm{H}}$ rearrangement is closely associated with non-coding transcription, epigenetic modifications and the recruitment of chromatin remodelers across the $V_{H}$ region ${ }^{17,31,32,33}$. It has been widely assumed that these developmentally regulated alterations of chromatin across the lgh locus simply function to promote accessibility ${ }^{32}$. Here we propose that epigenetic modifications of the chromatin fiber across the Igh locus generate, in a developmentally regulated fashion, a dispersed population of weak, reversible cross-links, which, in turn, induce phase-separated gel droplets, or segregated regions of crossbridged chromatin fiber (Fig. 6) ${ }^{4,34}$. Such cross-links would first induce a gel droplet comprising the $D_{H^{-}} J_{H}$ loop domain, thereby facilitating $D_{H}-J_{H}$ encounters within the loop domain and simultaneously functioning as an insulator to prevent the $D_{H}$ and $J_{H}$ segments from prematurely interacting with $V_{H}$ segments located outside the $D_{H}-J_{H}$ loop domain. Upon the formation of the $D_{H} J_{H}$ segment, epigenetic marks would be erased and non-coding transcription halted to allow the cross-links to dissociate and the gel droplet to dissolve. This step would lead to the disassembly of the $D_{H} J_{H}$ loop domain, formation of a de novo loop domain containing $\mathrm{V}_{\mathrm{H}}$ and $\mathrm{D}_{\mathrm{H}} \mathrm{J}_{\mathrm{H}}$ elements, induction of epigenetic modifications and non-coding transcription across the $V_{H}$ region, the formation of a phase-separated gel droplet comprising the $V_{H}-D_{H} J_{H}$ loop domain, and culminating in the $\mathrm{V}_{\mathrm{H}}-\mathrm{D}_{\mathrm{H}} \mathrm{J}_{\mathrm{H}}$ rearrangement. In conclusion, we propose that gelation within chromatin loop domains, induced by epigenetic modifications, chromatin remodelers and noncoding transcription, introduces the facilitation and hindrance modes in $V_{H^{-}} D_{H} J_{H}$ and $D_{H^{-}} J_{H}$ encounters thereby orchestrating a stepwise, ordered process for antigen receptor rearrangement. At the same time, the proximity of the Igh locus to the sol/gel boundary in the phase space, and the resulting propensity to switch between solid and liquid states, offers a tradeoff between stability and responsiveness in the generation of antigen receptor diversity. 


\section{Methods}

\section{Construction of tandem arrays of MUT-TET repressor binding sites}

WT-TET repressor binding sites were composed of TCTCTATCAGTGATAGGGA DNA sequences. MUTTET repressor binding sites contained the TCTCCGTCAGTGACGGGGA DNA sequence. DNA fragments containing four randomly repeated TET-repressor binding sites, each flanked by 10 bp random DNA sequence, were generated from synthetic oligonucleotides and inserted into a plasmid backbone using Nhe1, Xba1 and Pst1 restriction sites. Tandem arrays containing 320 copies of the MUT-TET binding sites were generated by sequential cloning and expansion of the number of repeats as described previously (Lau et al., 2003).

\section{Constructs and Retrovirus Production}

To generate Sc-Mut-TetR(B)-SNAP, mutant TetR was generated by site directed mutagenesis of TetREGFP to replacing glutamic acid residues at position 37 to alanine and proline at position 39 to lysine (Lucas et al. 2014). The above-generated vector was digested by Mfel and Sall and ligated with a Mfeland Sall-digested gBlock segment containing $\mathrm{SG}_{4}$ linker, Mutant-TetR, and SNAP tag to obtain Sc-MutTetR(B)-SNAP. Vectors were purified by $\mathrm{CsCl}$ gradient equilibrium centrifugation and transfected into 293T cells along with retroviral packaging vectors by calcium phosphate transfection. Medium was replaced the following morning, and viral supernatant was harvested 1 day later and stored at $-80 \mathrm{C}$ until used.

\section{Cell Culture and Transduction}

B lineage cells were grown in OptiMEM media with 10\% FCS and $1 \%$ Pen-Strep-Glutamine (Life Technologies) in the presence of IL7 and SCF. Supernatant from IL7-expressing cell lines was added at a concentration previously determined to promote B cell growth. Cells were incubated at $37^{\circ} \mathrm{C}$ under $5 \%$ CO2. TetR-EGFP and Sc-Mut-TetR-SNAP were co-infected and after two days cells were labeled with either SNAP-cell 647-SiR or SNAP-cell TMR-Star according to manufacturer's (NEB) instructions. For the 
locus contraction experiments, cells were treated with $10 \mu \mathrm{m}$ STI-571 for 36 hours before performing 3D DNA FISH or live imaging. 3D DNA FISH was performed as described before (Jhunjhunwala et al.).

\section{Imaging}

Imaging was performed under normal growth conditions using phenol red-free media (Carlton et al., 2010). Cells were plated on Fluorodish poly(D-lysine) coated plates (World Precision Instruments) at least 2 hours before imaging. Cells were imaged using a 100X 1.4NA oil immersion objective on an Applied Precision OMX microscope equipped with an EM-CCD camera. 20-30 Z sections were obtained with a spacing of $0.5 \mu \mathrm{m}$. Images were acquired at a rate of one stack every two seconds for a total of 200 total time points or one stack every 40 seconds for a total of 120 time points.

\section{Imaging Analysis}

An Applied Precision OMX microscope was used for live-cell microscopy. Data were deconvolved using Applied Precision SoftWorx software. The center of mass of Igh loci TetO probes was tracked at each time point using the "spots" feature of Bitplane's Imaris software. The "quality" threshold was manually adjusted for each cell so that the TetO probes were recognized at each time point. The "track duration" filter was used to remove incorrectly recognized spots.

\section{Modeling the chromatin fiber as a worm-like chain}

The $3 \mathrm{Mb}$ Igh locus was modeled as a bead-spring self-avoiding worm-like chain, using an open source Molecular Dynamics simulation platform LAMMPS (Plimpton, 1995). The chromatin fiber was simulated using, as parameter values, $50 \mathrm{bp} / \mathrm{nm}$ for the packing density of the fiber and $14 \mathrm{~nm}$ for the diameter of the bead, resulting in 700 bp per bead and a total of 4298 beads in the lgh locus. These values are consistent with estimates derived from experiments (Jhunjhunwala et al., 2008; Sanborn et al., 2015; Ou et al., 2017). The known genomic locations of individual $\mathrm{V}_{H}, \mathrm{D}_{\mathrm{H}}, \mathrm{J}_{\mathrm{H}}$ and CTCF elements determined the beads on which these elements were positioned in the simulations. The consecutive beads were 
connected by harmonic springs. The repulsive part of the Lennard-Jones potential was used to account for the physical volume occupied by each bead. The choice of parameters allowed occasional chain selfcrossing, mimicking a moderate topoisomerase activity. The persistence of the chain was modeled through an angle potential which gave rise to a persistence length of approximately $22 \mathrm{~nm}$. The dynamics of the chain was simulated using LAMMPS fix commands that perform Langevin dynamics according to the Langevin equation.

\section{Simulating a spectrum of chromatin configurations}

The hierarchy of the simulated polymer models (Fig. 5a) included a simple unstructured chromatin chain, a one-loop configuration of the chain, a two-loop configuration, and a spatially confined chain mimicking the effect of an "averaged" multiple-loop configuration. The looped configurations were engineered by introducing harmonic spring-bonds between the beads that anchored the loops. The simulation of a spatially confined chain was performed by introducing a global spherical confining potential of diameter $0.8 \mu \mathrm{m}$ to enclose the otherwise unstructured chain. To obtain the spatial distance vs genomic distance relation, a statistical ensemble of 1000 chains was generated for each chromatin configuration.

\section{Simulating crosslinks with varying degree of reversibility}

Weak intra-chain interactions were modeled by introducing $5 \%$ equidistant crosslinkable sites into the simulation of the spatially confined chain (Fig. 5b; Supplementary Information). The crosslinkable beads could undergo pairwise binding/unbinding events. The degree of reversibility of the crosslinks was varied by tuning the lifetime of the crosslinks. In order to explore the exclusive effect of crosslinks, a control set of simulations was performed by replacing the global confining potential with periodic boundary conditions.

\section{First-passage time analysis of experimental and simulated $V_{H}-D_{H} J_{H}$ trajectories}

A potential encounter event was identified as a dip in the distance between the $V_{H}$ and $D_{H} J_{H}$ segments below a threshold value. The threshold value $r_{c}$ was determined by combining the value of the true 
interaction distance $r_{\text {int }}$ and the localization error $r_{\text {err }}$ through $r_{\mathrm{c}}=\left(r_{\text {int }}^{2}+r_{\text {err }}^{2}\right)^{0.5}$. The value of $r_{\text {int }}$ was set to be $30 \mathrm{~nm}$, and the value of $r_{\mathrm{err}}{ }^{2}$ was extracted from the control experiment on measurement error to be $0.015 \mu \mathrm{m}^{2}$. The time corresponding to the first dip provides an estimation for the first-passage time. The first-encounter times from experiment and simulation as a function of mean separation distance within the $V_{H^{-}} D_{H} J_{H}$ pair, as well as the distributions of the first-encounter times, were compared (Fig. $5 d$ ). The firstencounter times from experiment were fitted with a power-law relationship FPT $=\beta<r^{2 / a}$. The fit yielded $\alpha$ $=0.17$ and $\beta=10^{8} \mu \mathrm{m}^{-2 / \alpha} \mathrm{S}$. The value of $\alpha$ is close to $\alpha=0.13$ extracted from the fit of the MSD of STI-571 treated pro-B cells, thus validating the predicted scaling MFPT $\sim R^{2 / a}$.

\section{References}

1. Sedat, J. \& Manuelidis, L. A direct approach to the structure of eukaryotic chromosomes. Cold Spring Harbor Symp Quant Biol. 42, 331-350 (1977).

2. Lieberman-Aiden, E. et al. Comprehensive mapping of long-range interactions reveals folding principles of the human genome. Science 326, 289-293 (2009).

3. Nasmyth, K. Disseminating the genome: joining, resolving and separating siste chromatids during mitosis and meiosis. Annu Rev Genet 35, 673-745 (2001).

4. Hnisz, D., Shrivinas, K., Young, R. A., Chakraborty, A. K. \& Sharp, P. A. A phase separation model for transcriptional control. Cell 169, 13-23 (2017).

5. Alt, F. W., Zhang, Y., Meng, F. L., Guo, C. \& Schwer, B. Mechanisms of programmed DNA lesions and genomic instability in the immune system. Cell 152, 417-429 (2013).

6. Ji, Y. et al. The in vivo pattern of binding of RAG1 and RAG2 to antigen receptor loci. Cell 141, 419431 (201).

7. Teng, G. \& Schatz, D. G.. Regulation and evolution of the RAG recombinase. Adv Immunol 128, 1-39 (2015). 
8. Benner, C., Isoda, T. \& Murre, C. New roles for DNA cytosine modification, eRNA, anchors and superanchors in developing B cell progenitors. Proc Natl Acad Sci USA 112, 12776-12781 (2015).

9. Lin, S.G., Guo, C., Su, A., Zhang, Y. \& Alt, F. W. CTCF-binding elements 1 and 2 in the Igh intergenic control region cooperatively regulate V(D)J recombination. Proc Natl Acad Sci US. 112, 18515-1820 (2015).

10. Alt, F. W. et al. Ordered rearrangement of immunoglobulin heavy chain variable region segments. EMBO J 3, 1209-1219 (1984).

11. Guo, C. et al. CTCF-binding elements mediate control of V(D)J recombination. Nature 477, 424-430 (2011).

12. $\mathrm{Hu}$, et al. Chromosomal loop domains direct the recombination of antigen receptor genes. Cell 163, 947-959 (2015).

13. Kumari, G. \& Sen, R. Chromatin interactions in the control of immunoglobulin heavy chain gene assembly. Adv Immunol 128, 41-92 (2015).

14. Chowdury, D. \& Sen, R. Stepwise activation of the immunoglobulin heavy mu chain locus. EMBO J 20, $6394-403(2001)$

15. Chowdury, D. \& Sen, R. Regulation of immunoglobulin heavy chain rearrangements. Immunol Rev 200, 182-96 (2004).

16. Medvedovic, J. et al. (2013). Flexible long-range loops in the $V_{H}$ gene region of the lgh locus facilitate the generation of a diverse antibody repertoire. Immunity $39,229-244$.

17. Bossen, C. et al. The chromatin remodeler Brg1 activates enhancer repertoires to establish B cell identity and modulate cell growth. Nat Immunol 16, 775-784 (2015).

18. Weber, S. C., Spakowitz, J.A. Theriot, Bacterial chromosomal loci move subdiffusively through a viscoelastic cytoplasm. Phys Rev Lett 104, 238102- 238107 (2010).

19. Lucas, J. S., Zhang, Y., Dudko, O. K. \& Murre, C. 3D Trajectories adopted by coding and regulatoryDNA elements: First-passage times for genomic interactions. Cell 158, 339-352 (2014). 
20. Krueger, C., Berens, C., Schmidt, A., Schnappinger, D. \& Hillen, W. Single chain Tet transregulators. Nucl Acid Res 31, 3050-3056 (2003).

21. Krueger, M., Scholtz, O., Wisshak, S. \& Hillen, W. (2007). Engineered Tet repressors with recognition specificity for the tetO-4C5G operator variant. Gene 404, 93-100.

22. Lau I.F. et al. Spatial and temporal organization of replicating Escherichia coli chromosomes. Mol Microbiology 49, 731-743 (2003).

23. Krueger, M., Scholtz, O., Wisshak, S. \& Hillen, W. Engineered Tet repressors with recognition specificity for the tetO-4C5G operator variant. Gene 404, 93-100 (2007).

24. Plimpton, S. Fast parallel algorithms for short-range molecular dynamics. J Comp Phys 117, 1-19 (1995).

25. Jhunjhunwala, S. et al. The 3D structure of the immunoglobulin heavy-chain locus: implications for long-range genomic interactions. Cell 133, 265-279 (2008).

26. Guo, C., Gerasimova, T., Hao, H., Ivanova, I., \& Sen, R. Two forms of loops generate the chromatin conformation of the immunoglobulin heavy chain locus. Cell 147, 332-343 (2011).

27. de Gennes, P. Scaling Concepts in Polymer Physics (Cornell University Press, Ithaca: New York, (1979).

28. Harmon, T. S., Holehouse, A. S. Rosen, M. K. \& Pappu, R. V. Intrinsically disordered linkers determine the interplay between phase separation and gelation in multivalent proteins. eLife 6, e30294 (2017).

29. Mora, T. \& Bialek, W. Are biological systems poised at criticality? J Stat Phys 144, 268-302 (2011).

30. Teng, G. \& Schatz, D.G. Regulation and evolution of the RAG recombinase. Adv Immunol 128, 1-39 (2015).

31. Su, J. et al. Ezh2 controls B cell development through histone H3 methylation and Igh rearrangement. Nat Immunol 4, 124-131 (2003).

32. Chakraborty, T. et al. A 220 nucleotide deletion of the intronic enhancer reveals an epigenetic hierarchy in immunoglobulin heavy chain locus activation. J Exp Med 206, 1019-1027 (2009). 
33. Yancopoulos, G. D. \& Alt, F. W. Developmentally controlled and tissue-specific expression of unrearranged Vh gene segments. Cell 40, 271-281 (1985).

34. Sabari B.R. et al. Coactivator condensation at super-enhancers links phase separation and gene control. Science 361, 6400 (2018).

\section{Acknowledgements}

We thank Dr. Peter Geiduschek for insightful comments, suggestions and editing. We thank the members of the Murre laboratory and the Dudko group for stimulating discussions. We thank Jennifer Santini for help with imaging and image analysis. We thank Dr. William Bialek for insightful discussions. We thank Dr. Ned Wingreen for valuable insights and advice relating to the simulations. Our studies were supported by grants from the National Institutes of Health (U54DK107977) to O.K.D. and the National Institutes of Health (U54DK24230, AI082850, Al00880 and Al09599) to C.M. Y.Z. was supported by the Princeton Center for Theoretical Science the National Science Foundation (Grant PHY1607612), and the NSF Center for the Physics of Biological Function (PHY1734030).

\section{Authors contributions}

N.K. generated tandem arrays of mutant TET-repressor binding sites, cell lines that harbored MUT-TET operator binding sites, performed the live cell imaging experiments and measured the spatial distances as a function of elapsed time. Y.Z. analyzed the data, designed and performed simulations. J.S.L generated pro-B cell lines carrying tandem arrays of TET-operator binding sites. O.K.D. and C.M. supervised the study and wrote the paper. All authors discussed the results and commented on the manuscript.

\section{Competing interests}

The authors declare no competing financial interests. 


\section{Figure Legends}

Fig. 1. A Strategy to track $V_{H^{-}} D_{H} J_{H}$ motion in live $B$ cells. a, Schematic of the lgh locus showing the $V_{H}$, $D_{H}, J_{H}, C_{H}$ segments, intronic enhancer $(E \mu)$ and switch region repeats $(S \mu)$. $\mathbf{b}$, DNA sequences of wildtype and mutant TET operator binding sites. Nucleotides for which the mutant binding sites differ from that of WT-TET binding sites are indicated in red. c, Experimental strategy to design tandem arrays of mutant TET-operator binding sites. * indicates the junction of ligated DNA segments, each containing three MUTTET binding sites. $\mathbf{d}$, Construction of retroviral vectors to optimize the signal to noise ratio in pro-B cells transduced with virus expressing wild-type TET-EGFP and MUT-TET SNAP Tag.

Fig. 2. Tracking $V_{H}-D_{H} J_{H}$ Motion in Pro-B Cells. a, Live imaging of pro-B cells harboring $D_{H} J_{H} W T-T E T$ and $V_{H}$ MUT-TET alleles. Panels indicate pro-B cells carrying $D_{H} J_{H}$ WT-TET and $V_{H}$ MUT-TET alleles transduced with WT-TET GFP and MUT-TET SNAP-TAG. Top and middle panels show fluorescent foci in cells transduced with virus expressing WT-GFP and MUT-SNAP-TAG. Right-hand images show both green and red fluorescent foci representing labeled $D_{H} J_{H}$ and $V_{H}$ regions. b. Pro-B cell lines harboring tandem arrays of WT-TET GFP and MUT-TET SNAP-TAG were analyzed by 3D-FISH using fluorescently labeled $V_{H}$ and $D_{H} J_{H}$ probes (top panel) or obtained from fluorescently marked TET probes during live imaging (lower panel) for the same cell population. c, Distributions of spatial separations of the $V_{H}$ and $D_{H} J_{H}$ elements obtained from live pro-B cell imaging (black dots) compared to those obtained from 3DFISH measurements (orange dots). d, Experimental strategy to determine localization error. Localization error was determined by measuring the spatial distances separating red and green fluorescent signals.

Fig. 3. $V_{H^{-}} D_{H} J_{H}$ motion in $B$ cell progenitors is extremely confined. a, The spatial distances within $V_{H^{-}}$ $D_{H} J_{H}$ pairs fluctuates around respective average values that remain nearly constant over time. Colorcoding the distance trajectories according to their mean values reveals a de-mixing effect. Right panel: the corresponding probability distribution of $\mathrm{V}_{\mathrm{H}^{-}} \mathrm{D}_{\mathrm{H}} \mathrm{J}_{\mathrm{H}}$ distances. $\mathbf{b}$, The time-averaged radial MSD (colored lines) and the time-and-ensemble-averaged radial MSD (black line) for $V_{H^{-}} D_{H} J_{H}$ motion. c, Time-and- 
ensemble-averaged MSD of relative genomic motion for inter-chromosomal $D_{H} J_{H}-D_{H} J_{H}$ pair (green) and intra-chromosomal $V_{H^{-}} D_{H} J_{H}$ pair (red) before (pale lines) and after (bright lines) measurement-error correction. $\mathbf{d}$, VAFs for the $\mathrm{V}_{\mathrm{H}}-\mathrm{D}_{\mathrm{H}} \mathrm{J}_{\mathrm{H}}$ motion exhibit anti-correlations indicative of a push-back from the environment; the value at the dip approaches the theoretical limit $(-0.5)$ of extreme confinement. The collapse of the VAF curves upon rescaling of the time axis (right panel) indicates self-similarity of motion.

\section{Fig. 4. Molecular dynamics simulations capture structural and dynamic properties of the Igh locus.}

a, The mean spatial separation as a function of the genomic distance between the $D_{H} J_{H}$ element and the $\mathrm{V}_{\mathrm{H}}$-repertoire from FISH measurements (triangles) and from MD simulations of a hierarchy of polymer configurations (lines). A multiple-loop chromatin configuration, or, equivalently, a global spatial confinement, reproduces the experimentally observed plateau. $\mathbf{b}, \mathrm{A}$ snapshot from MD simulations of the bead-spring model of the Igh locus with cross-linkable sites. c, The insertion of crosslinks across the Igh locus in the simulations reproduces the experimentally observed de-mixing of the trajectories.

Fig. 5. Modeling the Igh locus as a weak gel reproduces $V_{H^{-}} D_{H} J_{H}$ first-encounter times. a, Tuning the lifetime of crosslinks results in a systematic change in the slope (a) of the MSD. Short-lived bonds (lifetime $\sim 1 \mathrm{~min}$ ) yield the best agreement between MSD from experiment and simulations. $\mathbf{b}$, Representative trajectories of the spatial distance within individual $\mathrm{V}_{\mathrm{H}}-\mathrm{D}_{\mathrm{H}} \mathrm{J}_{\mathrm{H}}$ pairs in STI-571 treated cells. Red circles indicate potential first encounters. The corresponding encounter times are within an hour for $V_{H}$ and $D_{H} J_{H}$ segments that were in close spatial proximity at the start of imaging. $\mathbf{c}$, Representative trajectories of $\mathrm{V}_{\mathrm{H}^{-}}$ $D_{H} J_{H}$ spatial distances from the MD simulations of the lgh locus modeled as a weak gel. $\mathbf{d}$, First encounter times as a function of mean spatial separation from experimental and simulated $V_{H}-D_{H} J_{H}$ trajectories (left). The distributions of the first-encounter times from experiment and simulation (right).

Fig. 6. V(D)J Recombination orchestrated by gelation and proximity to the sol-gel phase transition. (I) Igh locus harboring $\mathrm{D}_{\mathrm{H}}, \mathrm{J}_{\mathrm{H}}$ and $\mathrm{V}_{\mathrm{H}}$ regions in a germ-line configuration, with no loop domains or cross- 
links. (II) A chromatin loop domain containing $D_{H}$ and $J_{H}$ regions is formed, anchored by the CTCF sites flanking the $D_{H}$ elements and the CTCF sites in the super-anchor. (III) Weak crosslinks are established across the loop domain containing $D_{H}$ and $J_{H}$ regions. The resulting gel-droplet, which is phase-separated from the rest of the locus, facilitates encounters between $D_{H}$ and $J_{H}$ elements and hinders encounters involving $\mathrm{V}_{\mathrm{H}}$ elements. (IV) The CTCF sites are inactivated and crosslinks are dissolved; the mobility of the newly formed $D_{H} J_{H}$ segment is increased. (V) Chromatin loops containing $D_{H} J_{H}$ and $V_{H}$ regions are formed, anchored by the CTCF sites across the $\mathrm{V}_{\mathrm{H}}$-cluster and the CTCF sites in the super-anchor. (VI) Cross-links across a loop are established, resulting in a new phase-separated gel-droplet that facilitates encounters between spatially close $V_{H}$ and $D_{H} J_{H}$ elements.

\section{Integrated supplementary information}

\section{Supplementary Figure 1. Extracting genomic motion from observed motion.}

The relative motion of the genomic segments was extracted from the observed motion by eliminating the effect of nuclear rotation and the measurement error. To ensure that the extracted motion of the genomic segments was unaffected by the extraction procedure, two independent approaches, both involving no adjusting parameters, were utilized and demonstrated to yield nearly identical results across short (left) and long (right) time scales.

\section{Supplementary Figure 2. Tracking $V_{H^{-}} D_{H} J_{H}$ Motion in Live B Cell Progenitors Reveals a Spectrum of Dynamic yet Stable Chromatin Configurations.}

Temporal trajectories of $\mathrm{V}_{\mathrm{H}^{-}} \mathrm{D}_{\mathrm{H}} \mathrm{J}_{\mathrm{H}}$ spatial distances, color-coded according to their mean values, reveal a de-mixing effect, visualized as the "rainbow" pattern, whereby the distances for the individual $V_{H}-D_{H} J_{H}$ pairs fluctuate around their respective mean values that remained nearly constant for at least an hour.

Supplementary Figure 3. Mean squared displacement of inter-chromosomal motion is subdiffusive. Time-averaged radial MSD (colored lines) and the time-and-ensemble-averaged radial 
MSD (black line) for the inter-chromosomal $\left(D_{H} J_{H}-D_{H} J_{H}\right)$ motion in pro-B cells. Both types of MSD are subdiffusive $(\alpha<1)$ and are characterized by the similar values of the anomalous scaling exponent $\alpha$ (the slope) and the anomalous diffusion coefficient $D$ (the vertical offset). The short horizontal black line indicates the mean measurement error.

\section{Supplementary Figure 4. The mean spatial distance between $D_{H} J_{H}$ and $V_{H}$ averaged over 1000} explicit eight-loop configurations reproduced the experimentally observed plateau. Individual eightloop configurations were engineered by anchoring the CTCF site at the superanchor to 7 randomly selected CTCF sites in the $\mathrm{V}_{\mathrm{H}}$ region and the most distal CTCF site. The mean spatial distance as a function of the genomic distance was computed from the ideal-chain model for individual configurations (gray lines) and averaged over all random looping configurations (black line).

Supplementary Figure 5. The exclusive effect of crosslinks on the genomic motion. The timeaveraged radial MSD (gray lines) and time-and-ensemble-averaged radial MSD (colored lines) of the relative motion between the marked $V_{H}$ and $D_{H} J_{H}$ elements in the set of simulations with varying degree of reversibility of crosslinks and with the periodic boundary condition.

Supplementary Figure 6. Representative $V_{H}-D_{H} J_{H}$ trajectories across long time-scales. Potential firstencounter events are marked with red circles. The estimated FPTs are on the timescale of seconds to hours for the $V_{H}$ and $D_{H} J_{H}$ elements that were found in a close spatial proximity at the start of imaging.

\section{Supplementary Figure 7. Mean spatial distances and mean first-encounter times from the} molecular dynamics simulations for a hierarchy of the chromatin topologies. a, Chromatin loops bring genomically distant segments to spatial proximity. b, Looped configurations of chromatin reduce the mean first-encounter times between the $D_{H} J_{H}$ element and $V_{H}$ elements from tens of hours (left) down to biologically relevant timescales (seconds to a few hours, right). c, Mean first-encounter times as a function 
of the mean $\mathrm{V}_{\mathrm{H}}-\mathrm{D}_{\mathrm{H}} \mathrm{J}_{\mathrm{H}}$ spatial distance for the hierarchy of chromatin topologies. The best fit results in the slope of 4 , confirming the predicted scaling MFPT $\sim R^{2 / a}(\alpha=0.5$ in the simulations $)$.

\section{Theory and Modeling}

\section{Extracting genomic motion from observed dynamics}

The observed apparent relative motion of the genomic segments is a result of a superposition of the true relative motion of the genomic segments, the rotation of the cell and nucleus, and measurement error. We developed two independent procedures to extract the true genomic motion from the observed apparent dynamics. In the first procedure, we eliminated the rotational motion by taking advantage of the availability of pairwise $V_{H}-D_{H} J_{H}$ and $D_{H} J_{H}-D_{H} J_{H}$ spatial distances, which are unaffected by rotation, and performing the MSD analysis on the distance trajectories $r(t)$ as MSD $=\left\langle(r(t)-r(t+\tau))^{2}\right\rangle$. As the changes in distances reflect the changes in the radial direction of the true relative motion in three dimensions (3D), we refer to the MSD calculated this way as "radial MSD". It can be shown that, under the condition $|r(t)-r(t+\tau)| \ll<$ $r(t)$, the radial MSD from the observed distance trajectories, radMSD ${ }^{\text {observed }}$, is equal to $1 / 3$ of the MSD of the true relative motion in $3 D, M S D^{\text {genomic }}$, plus measurement error, $M S D^{\text {err }}$. The factor $1 / 3$ reflects the fact that the radial motion represents 1 out of 3 degrees of freedom of the full 3D motion. The MSD of the genomic motion between segments in $3 D$ is then

$$
\mathrm{MSD}^{\text {genomic }}=3 \text { radMSD }{ }^{\text {observed }}-\mathrm{MSD}^{\mathrm{err}} .
$$

Eq. (1) gives the MSD of the true relative motion for each of the two pairs of genomic segments, the intra-chromosomal $V_{H}-D_{H} J_{H}$ pair and the inter-chromosomal $D_{H} J_{H}-D_{H} J_{H}$ pair. We assessed the contribution of the measurement error, $\mathrm{MSD}^{\mathrm{err}}$, by performing a control experiment in which a red and a green markers were inserted in the same genomic region, so that any observed relative motion between these markers would be due to measurement error only. The MSD ${ }^{\text {err }}$ was calculated using the standard formula, $\left\langle(\boldsymbol{r}(t)-\boldsymbol{r}(t+\tau))^{2}\right\rangle$, where $\boldsymbol{r}(t)$ is the displacement 
vector between the two markers in the control experiment. We found the value of the $\mathrm{MSD}^{\mathrm{err}}$ to be approximately $0.02 \mu \mathrm{m}^{2}$, or an average localization error of $22 \mathrm{~nm}$ for the $x$ - and $y$ coordinates and $95 \mathrm{~nm}$ for the $z$-coordinate for each marker. The MSD plots in Fig. 4 in the main text were obtained following the above procedure.

In the second procedure, we used the fact that the genomic motion can be obtained by subtracting all other contributions away from the observed motion:

$$
\mathrm{MSD}^{\text {genomic }}=\mathrm{MSD}^{\text {observed }}-\mathrm{MSD}^{\text {rotation }}-\mathrm{MSD}^{\mathrm{err}} .
$$

The separation of individual contributions is valid as long as there is no correlation among the different types of motion and measurement error. Eq. (2) applies both to the $V_{H^{-}} D_{H} J_{H}$ and $D_{H} J_{H^{-}}$ $D_{H} J_{H}$ pairs. The $M S D^{\text {observed }}$ can be calculated directly from experimental data, MSD $=<(\boldsymbol{r}(t)-\boldsymbol{r}(t$ $+\tau))^{2}>$. The $\mathrm{MSD}^{\text {err }}$ was assessed through the measurement error control experiment described in the first procedure. The rotational contribution $M S D^{\text {rotation }}$ for the $V_{H^{-}} D_{H} J_{H}$ pair was estimated as follows. By substituting Eq. (1) into Eq. (2), we found:

$$
\mathrm{MSD}^{\text {rotation }}=\mathrm{MSD}^{\text {observed }}-3 \text { radMSD }{ }^{\text {observed }} \text {. }
$$

As each of the terms on the right-hand side can be obtained directly from the experimental data, we used Eq. (3) to evaluate the rotational contribution $M S D^{\text {rotation }}$ of the $D_{H} J_{H}-D_{H} J_{H}$ pair. We then utilized $M S D^{\text {rotation }}$ of the $D_{H} J_{H}-D_{H} J_{H}$ pair to estimate $M S D^{\text {rotation }}$ of the $V_{H^{-}}-D_{H} J_{H}$ pair by noting that, on average, the amplitude of the rotational motion of a pair of segments is proportional to the mean distance between the segments in that pair:

$$
\mathrm{MSD}^{\text {rotation }}\left(\mathrm{V}_{\mathrm{H}}-\mathrm{D}_{\mathrm{H}} \mathrm{J}_{\mathrm{H}}\right) \approx\left[\frac{r\left(\mathrm{~V}_{\mathrm{H}}-\mathrm{D}_{\mathrm{H}} \mathrm{J}_{\mathrm{H}}\right)}{r\left(\mathrm{D}_{\mathrm{H}} \mathrm{J}_{\mathrm{H}}-\mathrm{D}_{\mathrm{H}} \mathrm{J}_{\mathrm{H}}\right)}\right]^{2} \mathrm{MSD}^{\text {rotation }}\left(\mathrm{D}_{\mathrm{H}} \mathrm{J}_{\mathrm{H}}-\mathrm{D}_{\mathrm{H}} \mathrm{J}_{\mathrm{H}}\right)
$$

Here, $r\left(V_{H^{-}}-D_{H} J_{H}\right)$ and $r\left(D_{H} J_{H}-D_{H} J_{H}\right)$ are the mean distances between the segments of the corresponding pairs. We then obtained the true relative genomic motion of the $V_{H^{-}} D_{H} J_{H}$ pair by substituting the $M S D^{\text {rotation }}$ of the $\mathrm{V}_{\mathrm{H}^{-}}-\mathrm{D}_{\mathrm{H}} \mathrm{J}_{\mathrm{H}}$ pair found from Eq. (4) into Eq. (2).

Finally, as a self-consistency check, we compared the genomic motions of the $V_{H^{-}} D_{H} J_{H}$ pair extracted via these two procedures, Eq. (1) and Eq. (2). The two procedures yielded nearly 
identical results for the entire imaging time (Supplementary Figure 1), indicating that the extracted genomic motion is independent of the procedure used. Note that all the terms in Eq. (1) and Eq. (2) were calculated from the experimental data, and therefore both approaches involve no adjustable parameters.

\section{Modeling the chromatin fiber as a worm-like chain}

The mouse immunoglobulin heavy chain locus is located on chromosome 12, spanning the 3 Mb region between nucleotides $117,349,200$ and $114,341,024$. We modeled the lgh locus as a bead-spring self-avoiding worm-like chain, using an open source molecular dynamics simulation platform LAMMPS ${ }^{1}$. The parameter values for the simulated chromatin fiber were chosen by combining estimates reported in multiple experiments ${ }^{2,3,4}$. Specifically, we assumed the packing density of chromatin fiber to be $50 \mathrm{bp} / \mathrm{nm}$ and the diameter of the bead ( $d$ ) to be $14 \mathrm{~nm}$, leading to 700 bp per bead and a total of 4298 beads in the lgh locus. The known genomic locations of individual $V_{H}, D_{H}, J_{H}$ and CTCF elements determined the beads on which these elements were positioned in the simulations. Specifically, the fluorescently marked $V_{H}$ and $D_{H} J_{H}$ regions were positioned on beads 2712 and 467, respectively. The consecutive beads were connected through the harmonic spring potential $k(r-d)^{2}$ with the spring constant $k=25 k_{\mathrm{B}} T / d^{2}$, where $k_{\mathrm{B}}$ is the Boltzmann constant and $T$ was set to the room temperature, $300 \mathrm{~K}$. To account for the physical volume occupied by each bead, we introduced repulsive interactions between all pairs of beads except neighboring beads. The repulsion was modeled using the Lennard-Jones potential, $4 \varepsilon\left[(\sigma / r)^{12}-(\sigma / r)^{6}\right]$, with $\varepsilon=1 k_{\mathrm{B}} T$ and $\sigma=d / 1.12$. The potential was cut off and shifted to 0 at $r=d$ so that only the repulsive part of the potential was used in the simulation. The above choice of parameters allows occasional chain self-crossing, mimicking a moderate topoisomerase activity. The persistence of the chain was modeled through the angle potential a. $\cos (\theta)$ with $\theta$ being the angle formed between three successive beads. A proper choice of the 
prefactor in the bending energy, $a=2 k_{\mathrm{B}} T$, resulted in a persistence length of approximately 22 $\mathrm{nm}$.

The dynamics of the chain was simulated using the commands fix nve and fix langevin in LAMMPS, which performs Langevin dynamics according to the Langevin equation: $m d^{2} \boldsymbol{R}_{i} / d t^{2}=$ $-\xi d \boldsymbol{R}_{i} / d t-d U / d \boldsymbol{R}_{i}+\boldsymbol{f}$. In the equation, $m$ is the mass of the bead, $\boldsymbol{R}_{i}$ is the center position of the bead $i, \xi$ is the friction coefficient, $U$ includes all the interaction potentials described above, and $\boldsymbol{f}$ is the Gaussian-distributed noise with $<f_{\alpha}(t) f_{\beta}\left(t^{\prime}\right)>=2 k_{\mathrm{B}} T \xi \delta_{\alpha \beta} \delta\left(t-t^{\prime}\right)$, where $\alpha$ and $\beta$ denote the $x-, y$-, or $z$-component of $\boldsymbol{f}$. The mass of the bead was estimated by assuming that the density of chromatin fiber is comparable to that of water. The friction coefficient $\xi$ in the damping force term was calculated from Stokes-Einstein relation, $\xi=3 \pi \eta d$, where the dynamic viscosity $\eta$ was taken to be $1 \mathrm{~Pa} \cdot \mathrm{s}$ (close to that of honey). Note that even though the reported viscosity of nucleoplasm is much lower, the crowded environment of the nucleus and nonspecific adhesive interactions between molecules can result in a much higher effective viscosity for relatively large objects, such as the labeled chromatin segments. The above value of viscosity was chosen to best match the dynamical properties of segment motion observed in our experiments. To speed up the simulations, a larger value of the bead mass was used while keeping $\xi$ (or the diffusion coefficient) constant, which led to an increased velocity relaxation time and thus allowed a larger integration time step in the simulation. This method did not affect the accuracy of the simulations as the timescale of interest was much longer than the increased velocity relaxation time, i.e. the simulations were performed in the overdamped regime.

\section{Simulating a spectrum of chromatin configurations}

The hierarchy of the simulated polymer models included a simple unstructured chromatin chain, a one-loop configuration of the chain, a two-loop configuration, and a spatially confined chain mimicking an "averaged" multiple-loop configuration. The one-loop and two-loop configurations were engineered by introducing harmonic-spring bonds between the beads that anchored the 
loops, using the same bond potential as the harmonic potential used for the springs connecting consecutive beads. Specifically, in the one-loop configuration, the bond was introduced between a CTCF site in the superanchor region (bead 171) and a CTCF site upstream of the marked V region (bead 2723), thus enclosing the marked $V_{H}$ and $D_{H} J_{H}$ regions in the same loop. In the two-loop configuration, one bond was introduced between a CTCF site in the superanchor region (bead 171) and the most distal CTCF site (bead 4241), and another bond between the CTCF site adjacent to the marked $D_{H} J_{H}$ region (bead 550) and the CTCF site upstream of the marked $V_{H}$ region (bead 2723), thus separating the marked $V_{H}$ and $D_{H} J_{H}$ regions in different loops. The simulation of a spatially confined chain was performed by introducing a global spherical confining potential $k(d-r)^{2}$ to enclose the otherwise unstructured chain. Note that $r$ in the potential is the distance from the bead to the boundary of the sphere. The potential was only switched on when the bead was within a distance $d$ from the boundary, $r<d$. The spring constant $k$ was set to be the same as that of the springs connecting beads. The diameter of the confining sphere was set to be $0.8 \mu \mathrm{m}$.

The spatial distance vs genomic distance relation was obtained from a statistical ensemble of 1000 chains for each chromatin configuration. The spatial distances between the $D_{H} J_{H}$ bead and the rest of beads on the chain were calculated for each chain, averaged over the ensemble, and plotted as a function of genomic distance (Fig. 5a).

First-encounter times for the $D_{H} J_{H}$ segment and the $V_{H}$-repertoire in looped configurations

The effect of chromatin topology on the first-encounter times was examined quantitatively by performing Molecular Dynamics simulations of the hierarchy of the polymer configurations described above. Here, the packing density of chromatin fiber was set to be $130 \mathrm{bp} / \mathrm{nm}$ and the diameter of the bead to be $30 \mathrm{~nm}$, leading to 772 beads in the lgh locus and a persistence length of approximately $50 \mathrm{~nm}$. The marked $V_{H}$ and $D_{H} J_{H}$ regions were positioned on beads 487 
and 84. In the one-loop configuration, the bond was introduced between CTCF sites on beads 31 and 489. In the two-loop configuration, one bond was introduced between CTCF sites on beads 31 and 762, and another bond between CTCF sites on beads 99 and 489. The interaction distance, which signified a genomic encounter, was set to be $60 \mathrm{~nm}$.

To find the first-encounter time, the distance between $V_{H}$ and $D_{H} J_{H}$ beads was checked once every time interval equal to the velocity relaxation time of the beads. Once the $D_{H} J_{H}$ bead was within the interaction distance from a $V_{H}$ bead, the corresponding $V_{H^{-}} D_{H} J_{H}$ encounter time for that $\mathrm{V}_{\mathrm{H}}$ segment was recorded. To speed up the simulations, a larger value of the bead mass was used. The larger value of the bead mass and the chosen frequency of checking for a potential encounter only had minor effects on the first-encounter times: a control simulation showed that, upon decreasing the bead mass (and hence increasing the checking frequency) by a factor of 100 , the first-encounter times were only reduced by a factor of 2 . From the simulation, we found that the $D_{H} J_{H}$ segment can readily encounter $V_{H}$ segments located on different loops within biologically relevant timescales (Supplementary Figure 7).

\section{Simulating crosslinks with varying degree of reversibility}

The live-imaging data on the Igh locus dynamics indicated that the locus adopts a spectrum of stable configurations. To explore the possibility that weak intra-chain interactions could increase the spatial rigidity of the locus and hence stabilize the locus structure, we introduced $5 \%$ equally spaced crosslinkable sites (on beads $10,30,50, \ldots$ ) into the simulation of the spatially confined chain (Fig. 5b). The crosslinkable beads could undergo pairwise binding/unbinding events. The bound beads were subjected to a harmonic interaction potential $k_{b}\left(r-d_{b}\right)^{2}$ with the mean bond length $d_{b}=50 \mathrm{~nm}$ and a soft spring constant, $k_{b}=0.1 \mathrm{k}$. We performed a set of simulations with decreasing degree of reversibility of the crosslinks. The simulation without crosslinks (blue in Fig. 6a) is the same as the original simulation of the spatially confined chain. The simulations with reversible crosslinks were performed using the commands fix bond/create and fix 
bond/break in LAMMPS. Bonds were checked for creation once every $0.01 \mathrm{~s}$, and were created between unbound crossl-inkable bead pairs when their separations were smaller than $60 \mathrm{~nm}$. The created bonds were checked for breaking once every $1 \mathrm{~s}, 10 \mathrm{~s}$, or $100 \mathrm{~s}$ (orange, yellow, purple in Fig. 6a), and were broken if the separations between the bound beads were larger than $60 \mathrm{~nm}$. We estimated the bond lifetimes in these simulations to be about $10 \mathrm{~s}, 100 \mathrm{~s}$, and 1000 s, respectively. The simulation with irreversible crosslinks (green in Fig. 6a) was performed by only using the command fix bond/create. To keep the bond information updated, we replaced the original fix_bond_create.cpp file in LAMMPS MC package, which only counts the bonds once at the beginning of the simulation run, with a modified version ${ }^{7}$. Another set of simulations, which aimed to explore the exclusive effect of crosslinks (Supplementary Figure 5), was performed by replacing the global confining potential with periodic boundary conditions using a box size $0.6 \mu \mathrm{m}$. Each run in both sets of simulations with different degree of reversibility of crosslinks was repeated 10 times using a different random seed. The $x$-, $y$-, and $z$-coordinates of the crosslinkable beads in the chain were recorded once every $2 \mathrm{~s}$ for $4000 \mathrm{~s}$ to mimic the experimental protocol. To find the mean squared displacement of the relative motion between the marked $V_{H}$ and $D_{H} J_{H}$ beads (2712 and 467), the spatial distance between the two beads was calculated at each time point. The resulting distance trajectories were used in the MSD analysis (Fig. 6a). To enrich the statistics, we included in the MSD analysis the trajectories of the distances between beads with the same genomic separation as that of $V_{H}$ and $D_{H} J_{H}$ beads.

\section{First-encounter-time analysis of experimental and simulated $V_{H^{-}}-D_{H} J_{H}$ trajectories}

The two-color imaging strategy enabled a direct estimation of the $\mathrm{V}_{\mathrm{H}}-\mathrm{D}_{\mathrm{H}} \mathrm{J}_{\mathrm{H}}$ first-encounter times. A potential encounter event was identified as a dip in the distance between the $V_{H}$ and $D_{H} J_{H}$ segments below a threshold value. The threshold value $r_{\mathrm{c}}$ was determined by combining the value of the true interaction distance $r_{\text {int }}$ and the localization error $r_{\text {err }}$ through $r_{\mathrm{c}}=\left(r_{\text {int }}{ }^{2}+r_{\text {err }}^{2}\right)^{0.5}$. 
The value of $r_{\text {int }}$ was set to be $30 \mathrm{~nm}$, and the value of $r_{\mathrm{err}}{ }^{2}$ was extracted from the control experiment by averaging the squared distance between the two markers and found to be 0.015 $\mu \mathrm{m}^{2}$. Note that this value for $r_{\mathrm{err}}{ }^{2}$ is slightly larger than the value expected theoretically, $r_{\mathrm{err}}{ }^{2}=0.5$ $M S D^{\text {err }}$, likely due to the effect of chromatic aberration in the experiments. As a dip below the threshold is a potential encounter event, the time corresponding to the first dip provides a lower bound for the $\mathrm{V}_{\mathrm{H}}$ and $\mathrm{D}_{\mathrm{H}} \mathrm{J}_{\mathrm{H}}$ first-encounter time. On the other hand, as distance trajectories were recorded at a finite time interval, missed encounter events due to finite recording frequency may lead to an overestimation of the observed first-encounter times.

To investigate the relationship between the first-encounter times and the spatial distance, we generated a pool of contracted cells, in which the smaller spatial distances yielded richer statistics of the potential encounter events. Experimental $V_{H}-D_{H} J_{H}$ trajectories were recorded once every $2 \mathrm{~s}$ for $400 \mathrm{~s}$. To best mimic the experimental protocol, simulated $V_{H}-D_{H} J_{H}$ trajectories were recorded with the same frequency and duration. Measurement errors were randomly drawn from the trajectories obtained in the control experiment and added to the simulated $\mathrm{V}_{\mathrm{H}^{-}}-\mathrm{D}_{\mathrm{H}} \mathrm{J}_{\mathrm{H}}$ trajectories. To enrich the statistics, the trajectories of the distances between beads with the same genomic separation as that of $V_{H}$ and $D_{H} J_{H}$ beads were also included in the first-encounter time analysis.

The first-encounter times from experiment and simulation as a function of mean separation distance within the $\mathrm{V}_{\mathrm{H}}-\mathrm{D}_{\mathrm{H}} \mathrm{J}_{\mathrm{H}}$ pair, as well as the distributions of the first-encounter times, were compared (Fig. 6d). The first-encounter times from experiment were fitted with a power law relationship FPT $=\beta<r^{2 / a}$. As the finite length of trajectories (400 s) sets an upper limit for the observed FPTs, the parameters $\alpha$ and $\beta$ were found by minimizing the sum of the squares of the distance between individual data points and the fitted line along the $x$-axis. The fit yielded $\alpha=$ 0.17 and $\beta=10^{8} \mu \mathrm{m}^{-2 / \alpha} \mathrm{s}$. The value of $\alpha$ is in agreement with the subdiffusive exponent $\alpha=0.13$ extracted from the fit of the MSD of contracted cells, confirming the predicted scaling MFPT $R^{2 / a}$ 
bioRxiv preprint doi: https://doi org/10.1101/441444: this version posted October 12, 2018. The copyright holder for this preprint (which was not certified by peer review) is the author/funder, who has granted bioRxiv a license to display the preprint in perpetuity. It is made available under aCC-BY-NC-ND 4.0 International license. 


\section{REFERENCES}

1. Plimpton, S. Fast parallel algorithms for short-range molecular dynamics. J Com Phys 117, 1-19 (1995).

2. Jhunjhunwala, $S$ et al. The 3D structure of the immunoglobulin heavy-chain locus: implications for long-range genomic interactions. Cell 133, 265-279 (2008).

3. Sanborn, A.L et al. Chromatin extrusion explains key features of loop and domain formation in wild-type and engineered genomes. Proc Nat. Acad Sci USA 112 (47) E6456-E6465 (2015).

4. Ou, H.D et al. ChromEMT: Visualizing 3D chromatin structure and compaction in interphase and mitotic cells. Science 357, 6349-6358 (2017).

5. de Buyl, P. \& Nies, E. A parallel algorithm for step- and chain-growth polymerization in molecular dynamics. J Chem Phys 142, 134102 (2015).

6. Lucas, J.S., Zhang, Y., Dudko, O.K. \& Murre, C. (2014). 3D Trajectories adopted by coding and regulatory DNA elements: First-passage times for genomic interactions. Cell 158, 339-352 (2014). 

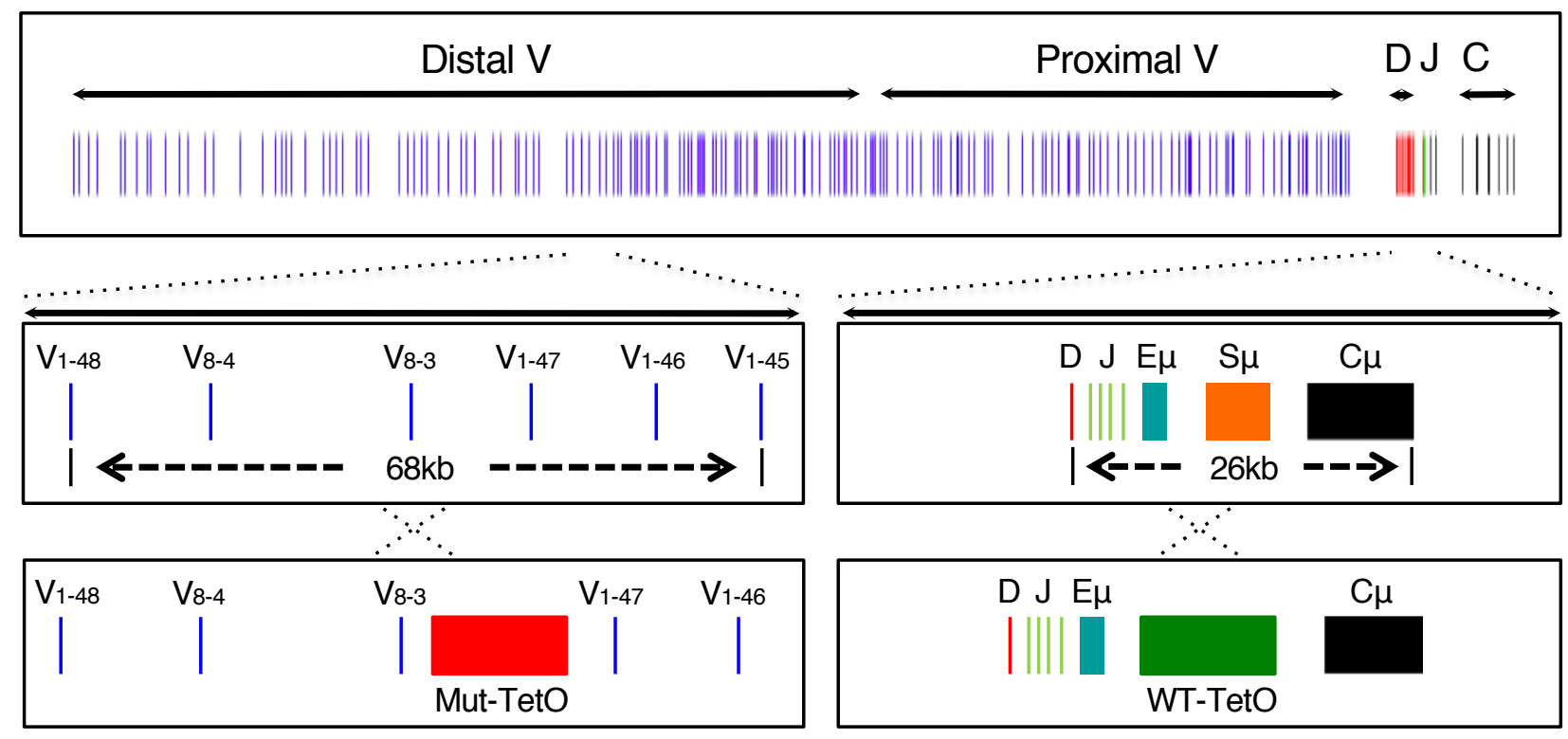

b

\begin{tabular}{|c|} 
wT-TetO \\
TCT CTATCA G TGATAG GGA \\
AGA GATAGT C ACTATC CCT \\
Mut-TetO \\
TCT CCGTCA G TGACGG GGA \\
AGA GGCAGT C ACTGCC CCT \\
\hline
\end{tabular}
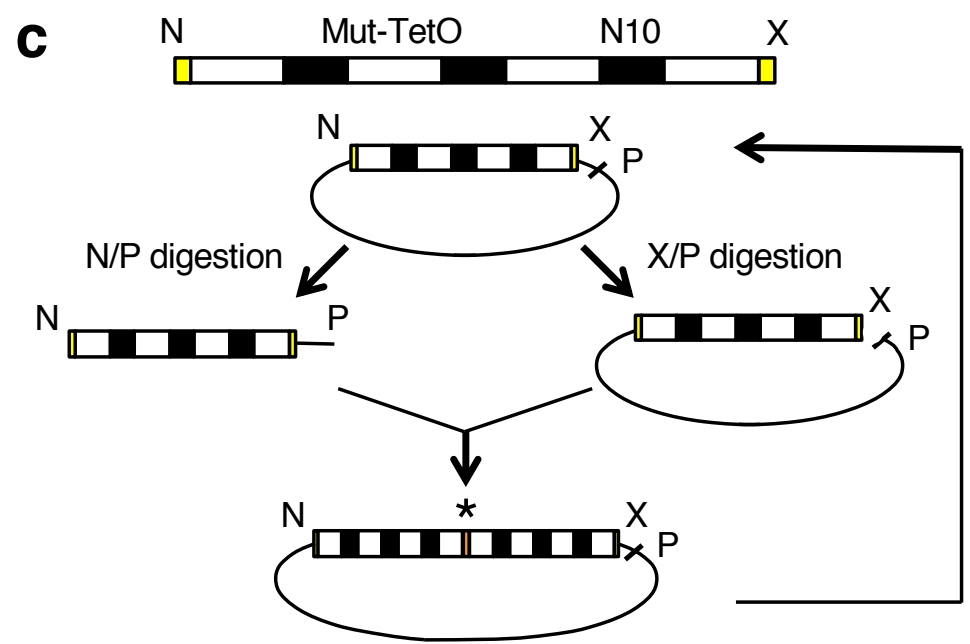

d

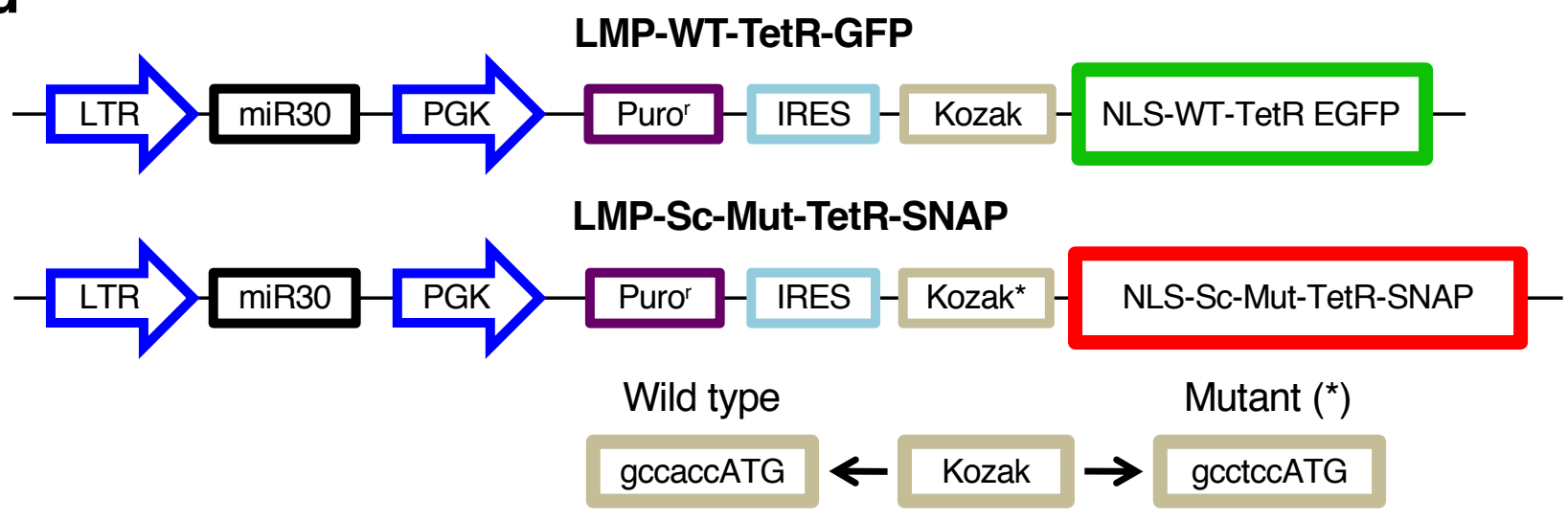


Figure 2

a
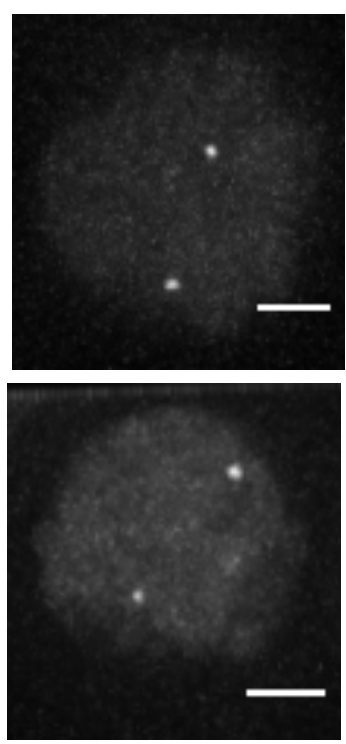

WT-TET GFP
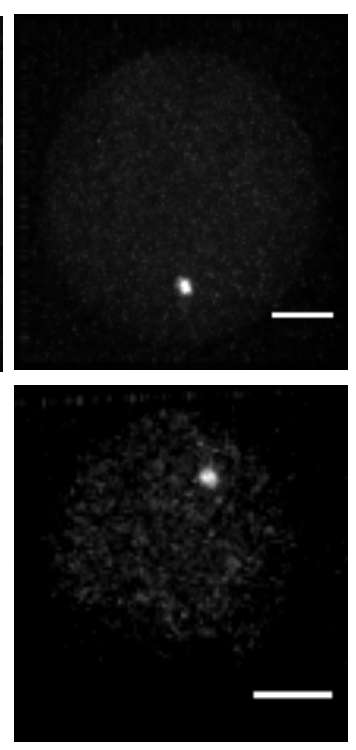

MUT-TET SNAP-TAG b
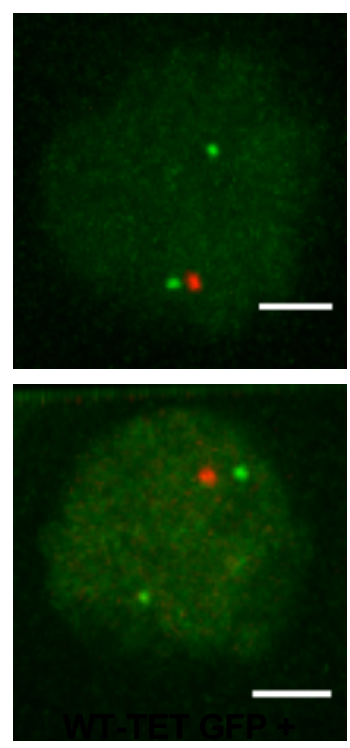

MUT-TET

SNAP-TAG
C

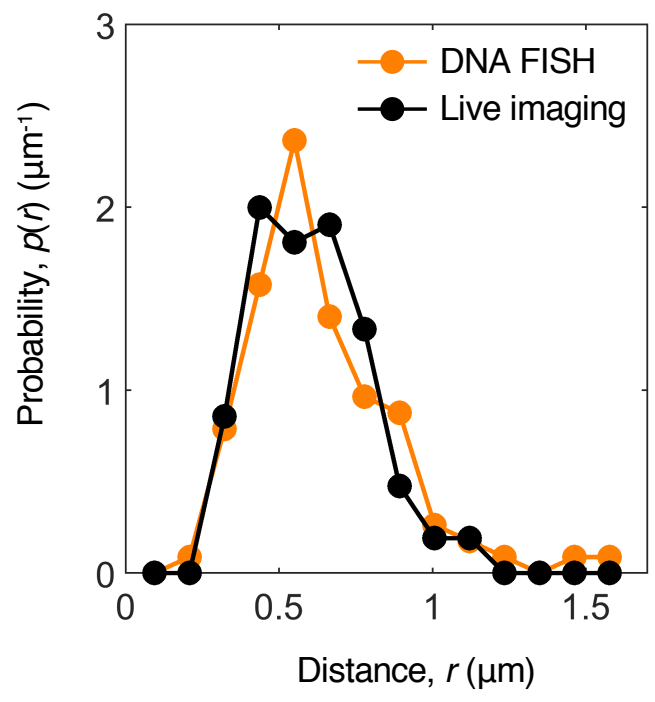

d

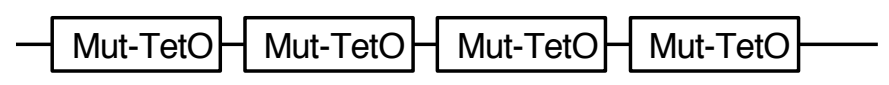

LMP-Mut-TetR-GFP

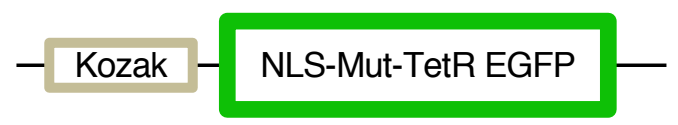

LMP-Sc-Mut-TetR-SNAP

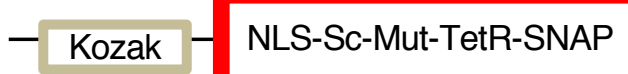

MUT-TET GFP + MUT-TET SNAP-TAG MUT-TET SNAP-TAG e MUT-TET GFP

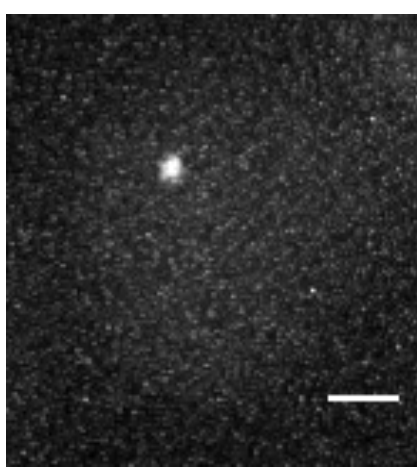

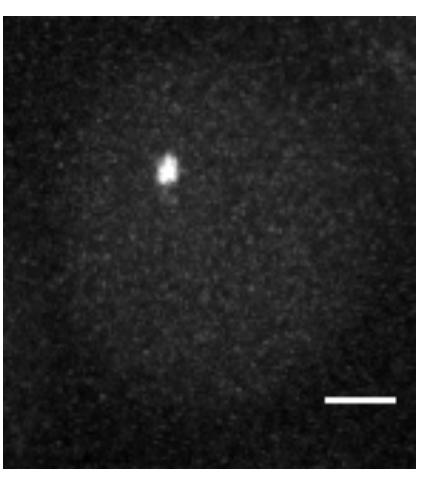

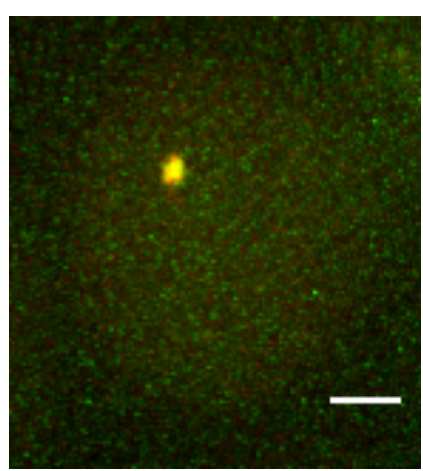


Figure 3

a

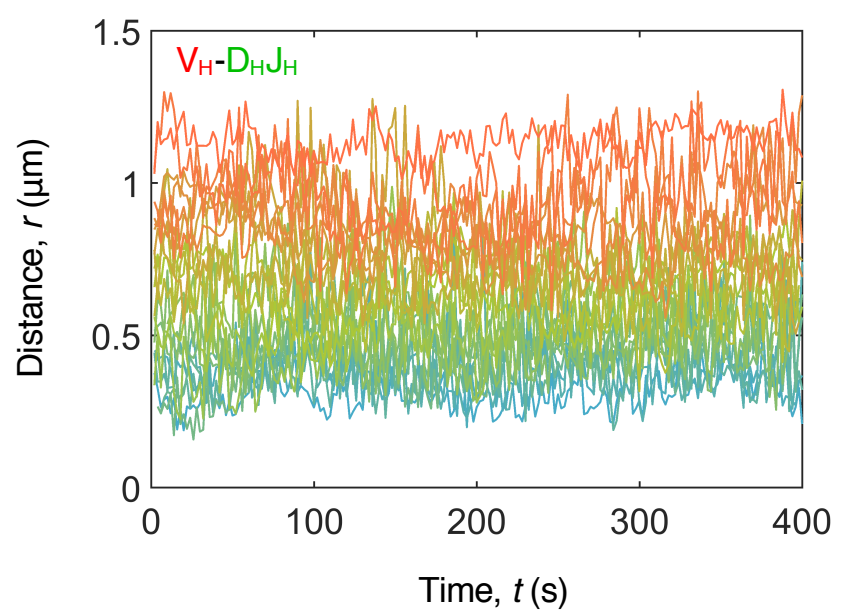

b

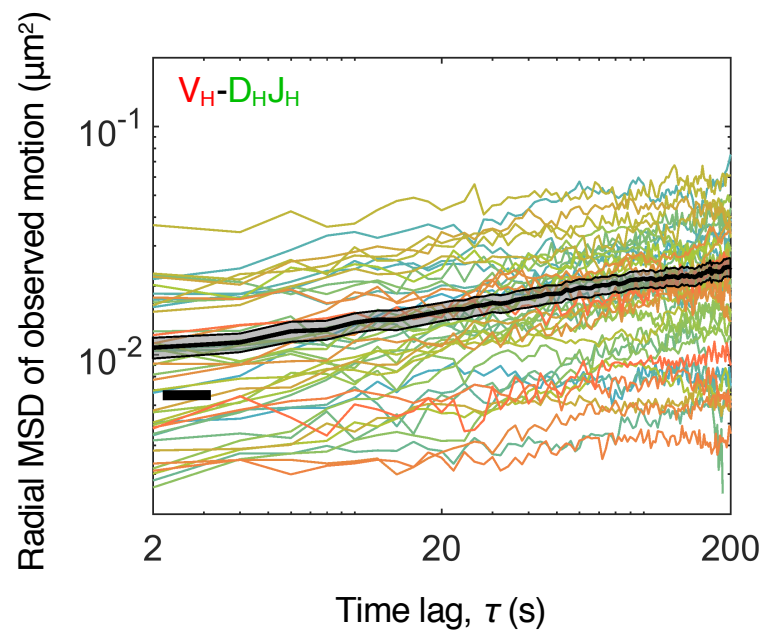

d

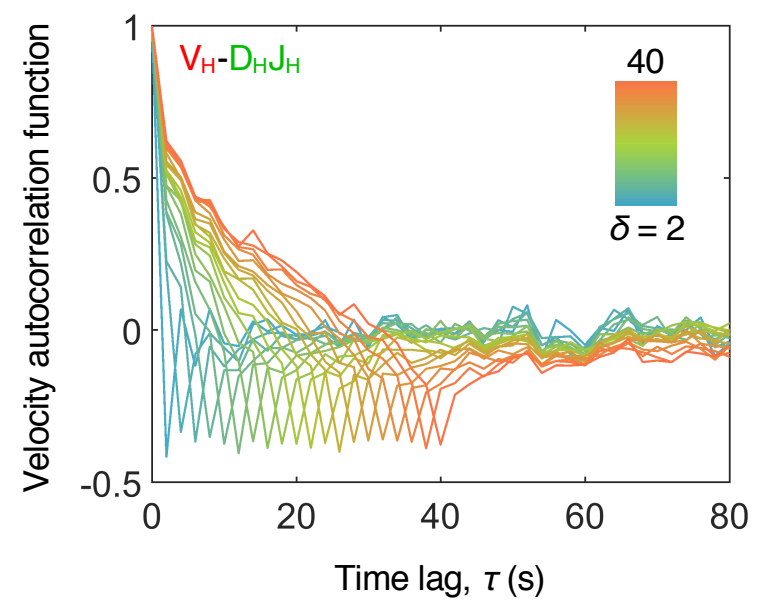

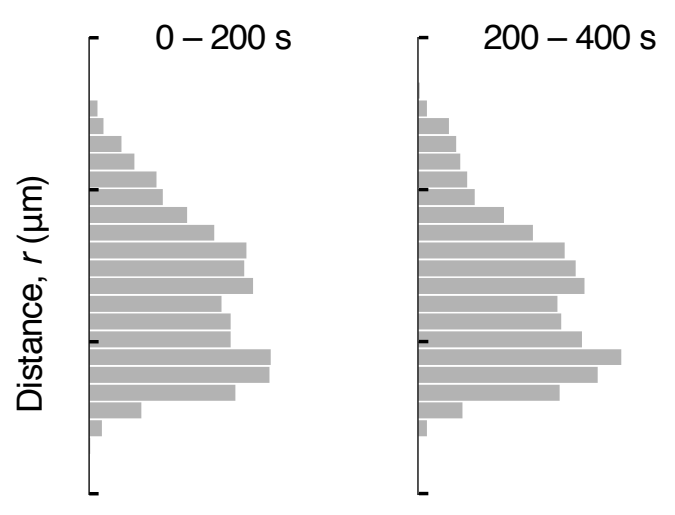

C
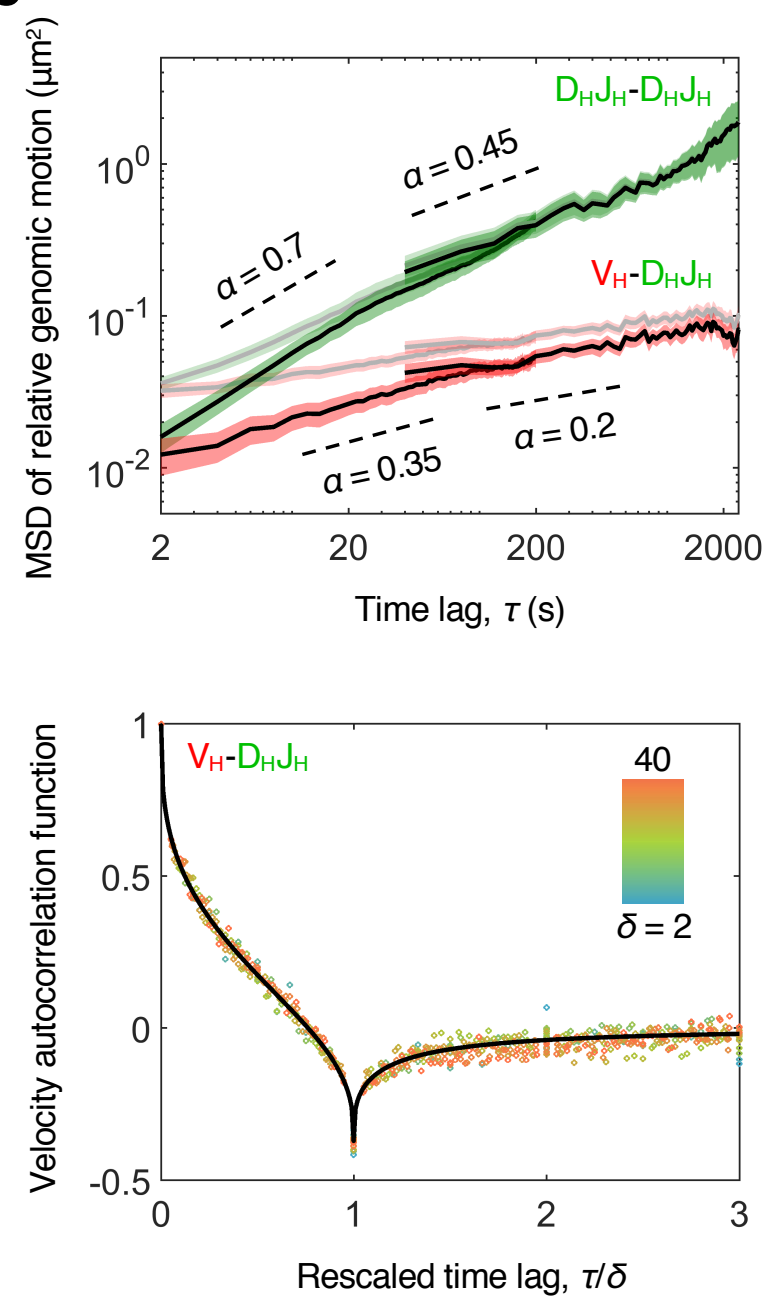
Figure 4

a Bead-spring polymer model

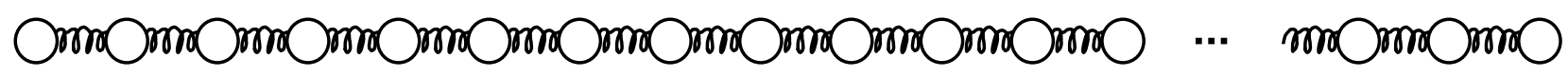
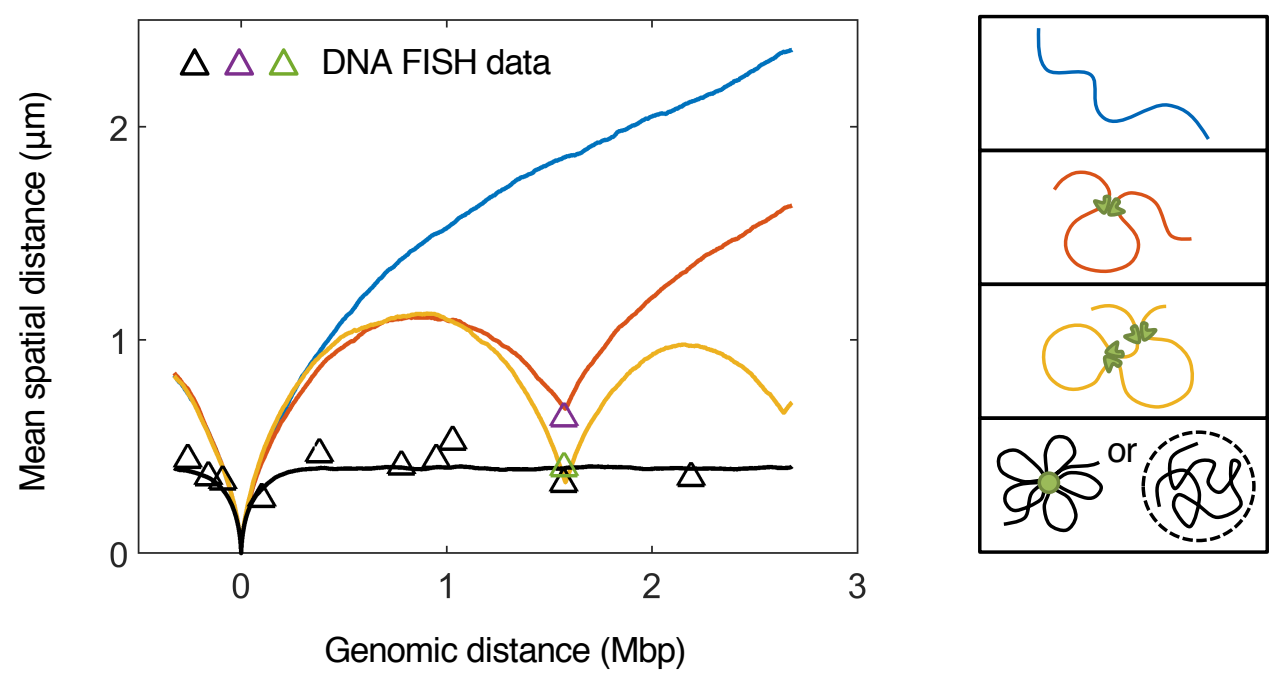

b

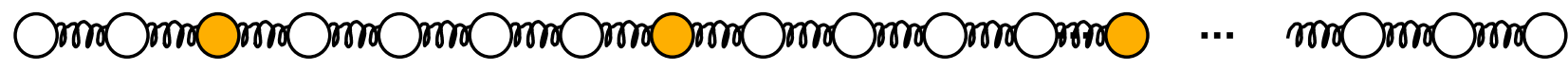
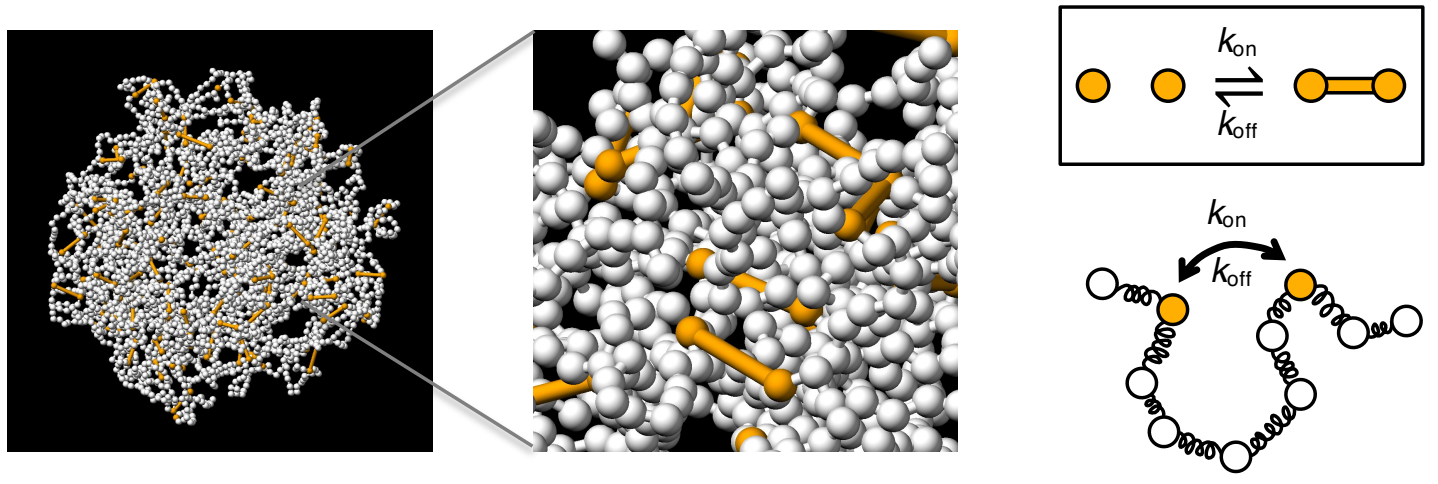

C

No crosslinks

Reversible crosslinks
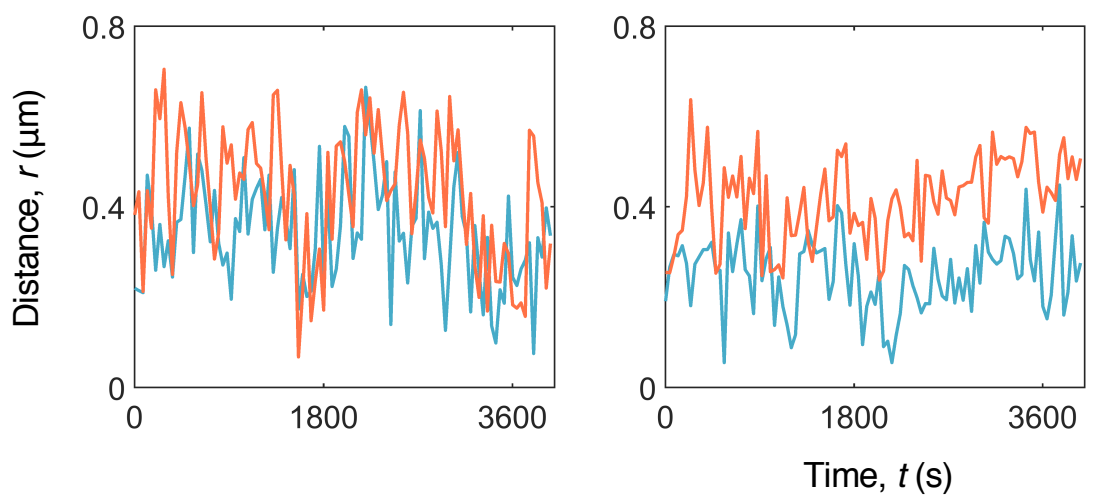

Irreversible crosslinks

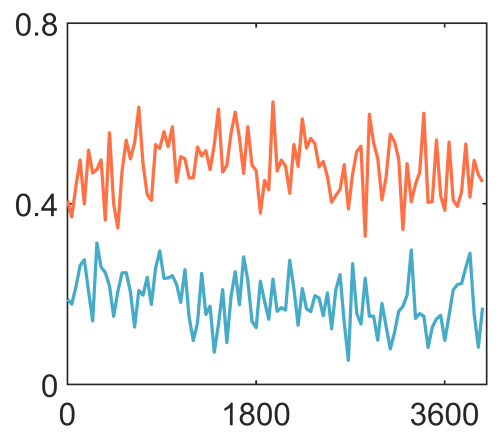



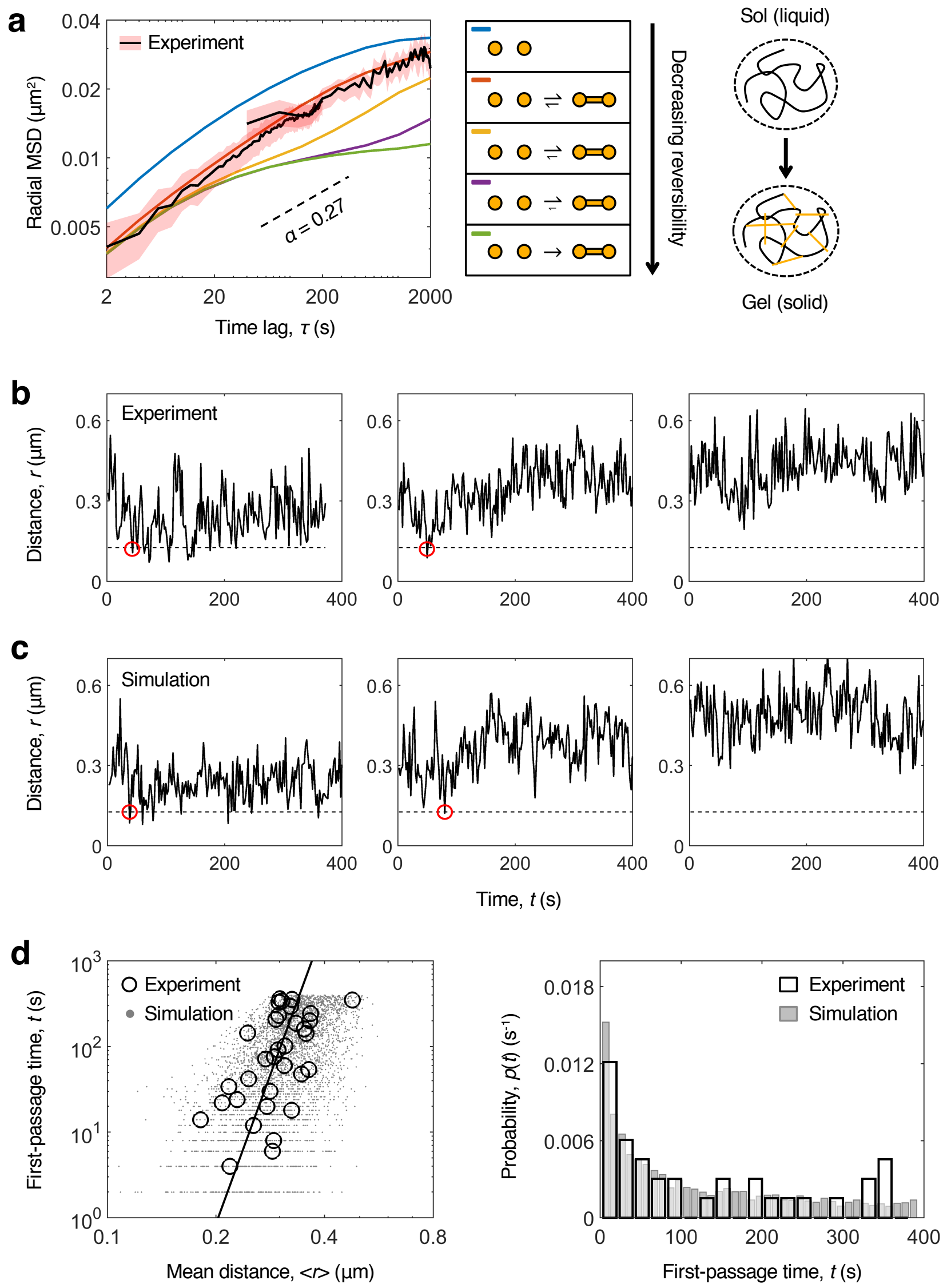
Figure 6

I

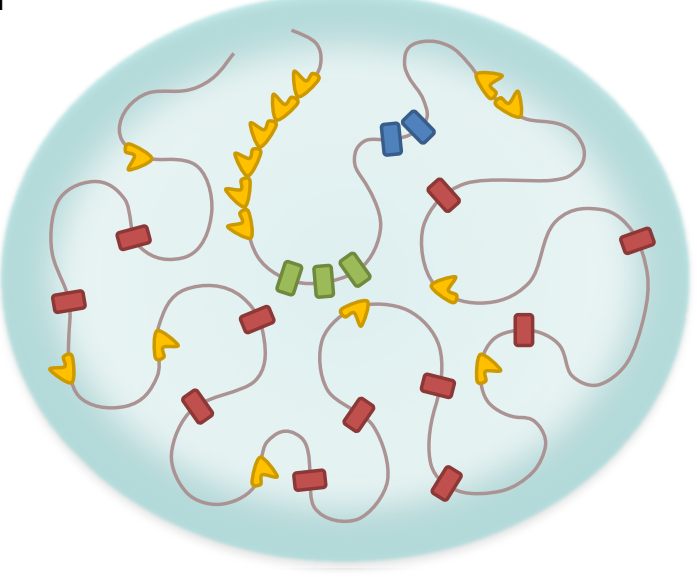

III

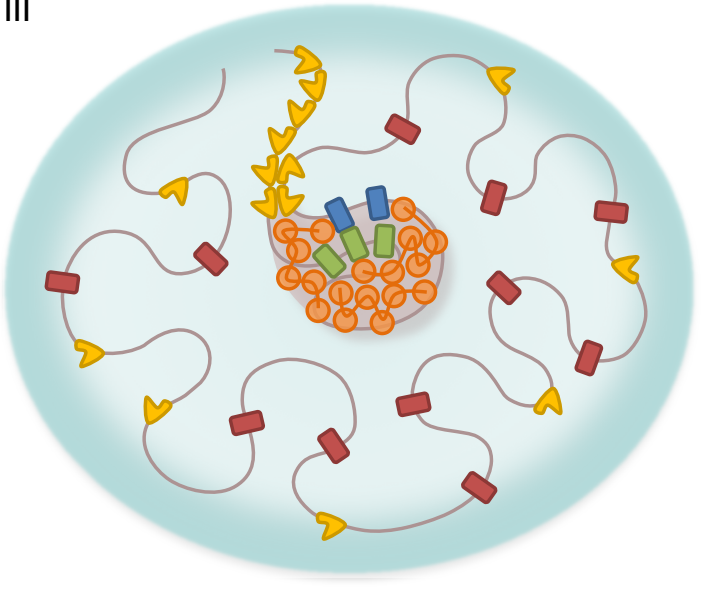

V

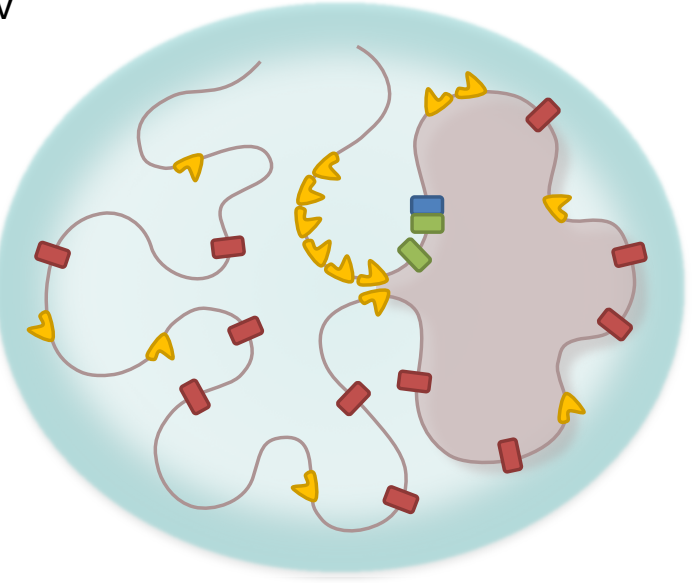

II

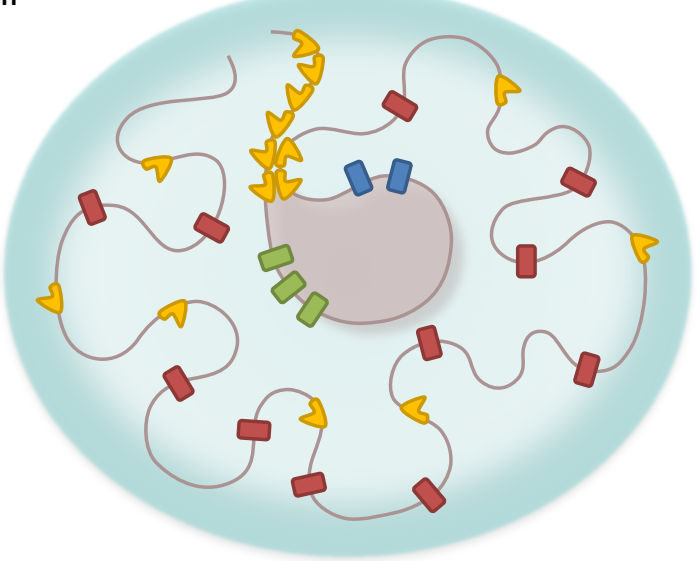

IV

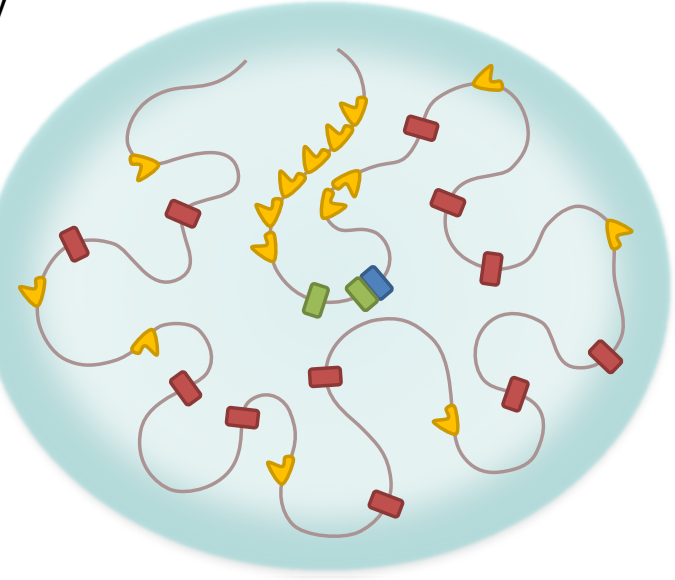

VI

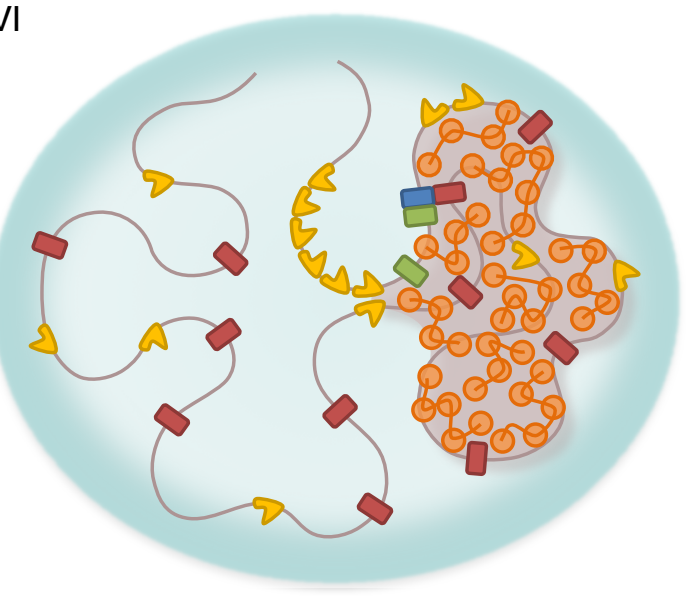

- $\mathrm{V}_{\mathrm{H}}$ segment $\square \mathrm{D}_{\mathrm{H}}$ segment $\square \mathrm{J}_{\mathrm{H}}$ segment $\triangle \mathrm{CTCF} \quad-$ crosslink 